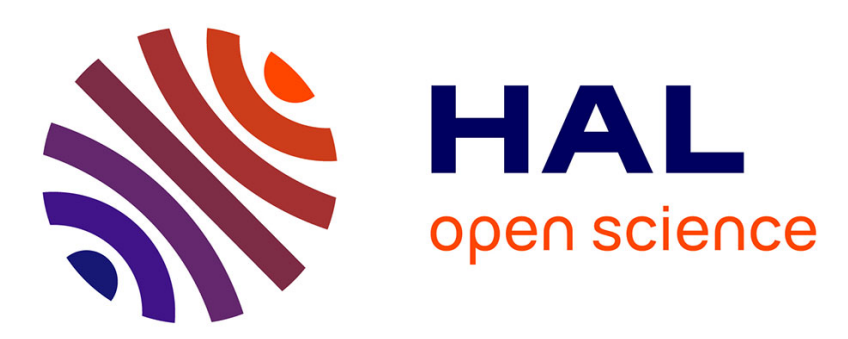

\title{
De la forêt primaire aux plantations coloniales : les deux ruptures politiques et socio-économiques de la côte est de Sumatra-Nord.
}

\author{
Daniel Perret
}

\section{- To cite this version:}

Daniel Perret. De la forêt primaire aux plantations coloniales : les deux ruptures politiques et socioéconomiques de la côte est de Sumatra-Nord.. Bulletin de l'Ecole française d'Extrême-Orient, 1994, 81 (1), pp.153 - 179. 10.3406/befeo.1994.2252 . halshs-01877456

\section{HAL Id: halshs-01877456 \\ https://shs.hal.science/halshs-01877456}

Submitted on 19 Sep 2018

HAL is a multi-disciplinary open access archive for the deposit and dissemination of scientific research documents, whether they are published or not. The documents may come from teaching and research institutions in France or abroad, or from public or private research centers.
L'archive ouverte pluridisciplinaire HAL, est destinée au dépôt et à la diffusion de documents scientifiques de niveau recherche, publiés ou non, émanant des établissements d'enseignement et de recherche français ou étrangers, des laboratoires publics ou privés. 


\section{De la forêt primaire aux plantations coloniales : les deux ruptures} politiques et socio-économiques de la côte est de Sumatra-Nord

\section{Daniel Perret}

\section{Citer ce document / Cite this document :}

Perret Daniel. De la forêt primaire aux plantations coloniales : les deux ruptures politiques et socio-économiques de la côte est de Sumatra-Nord. In: Bulletin de l'Ecole française d'Extrême-Orient. Tome 81, 1994. pp. 153-179;

doi : https://doi.org/10.3406/befeo.1994.2252

https://www.persee.fr/doc/befeo_0336-1519_1994_num_81_1_2252

Fichier pdf généré le 07/05/2018 


\title{
De la forêt primaire aux plantations coloniales
}

\author{
Les deux ruptures politiques et socio-économiques \\ de la côte est de Sumatra-Nord
}

\author{
Daniel PERRET
}

La côte nord-est de Sumatra est surtout connue pour avoir abrité, à partir de la seconde moitié du XIX ${ }^{e}$ siècle, la plus grande expérience capitaliste de l'époque coloniale sur l'archipel indonésien. En un temps relativement court à compter de 1863, de grandes plantations européennes et américaines se consacrant tout d'abord à la culture du tabac puis à celles de l'hévéa et du palmier à huile ont véritablement façonné en profondeur une région qui sera désignée dans la littérature coloniale avec les termes devenus fameux depuis de Oostkust (Côte Est) et de cultuurgebied (aire de plantations).

$\mathrm{Si}$ les spectaculaires bouleversements économiques, sociaux, politiques et culturels liés à cette expansion coloniale ont suscité plusieurs études, fort peu de travaux se sont véritablement intéressés à la situation précoloniale de la côte nord-est, en particulier celle du sultanat de Deli (qui verra s'installer les premières plantations occidentales) et de son arrière-pays.

Or, en faisant appel à des sources diversifiées, nous allons essayer de montrer que, au moins un demi-siècle avant les Occidentaux, une rupture s'est déjà produite avec le développement par les autochtones d'une véritable « aire de plantations », comparable sur de nombreux points à la cultuurgebied. C'est l'apparition de ces plantations indigènes ainsi que l'organisation socio-économique sur laquelle elles s'appuient que nous chercherons à reconstruire. Cet éclairage permet d'autre part de mieux comprendre les phénomènes d'adaptations et réactions des autochtones face aux bouleversements qui vont toucher la région à partir de 1863.

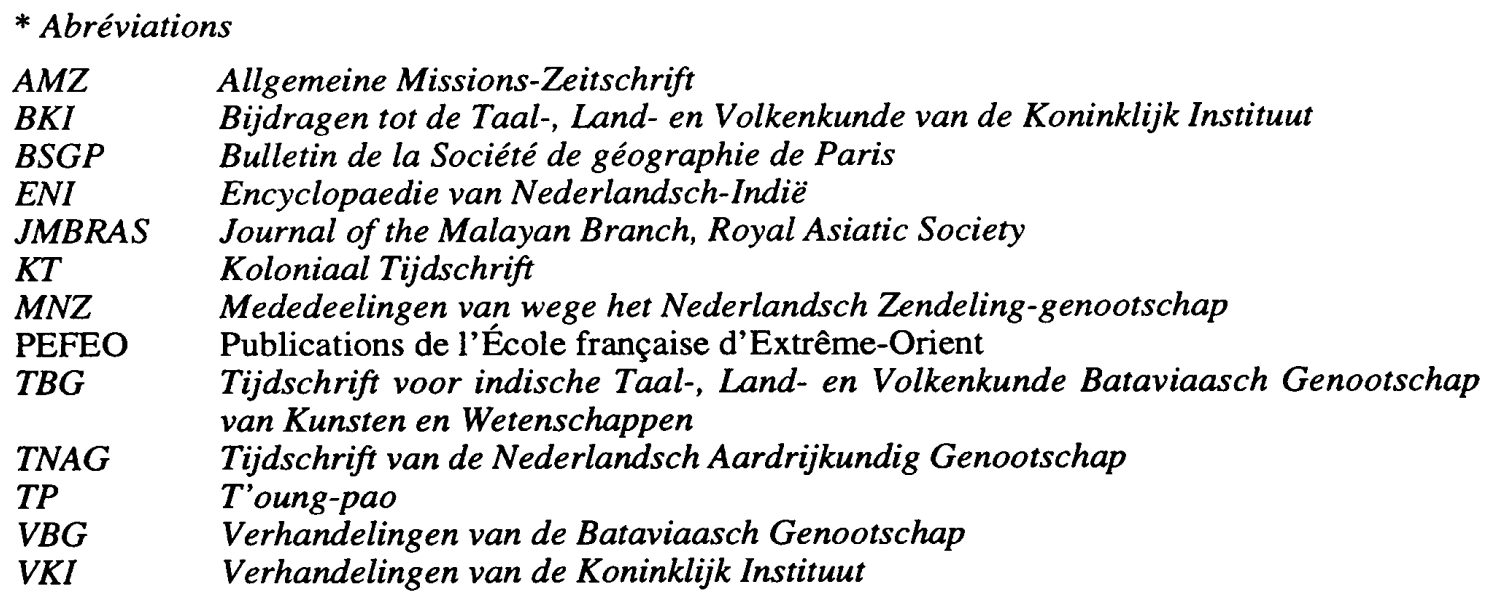




\section{Les premiers moments : cueillette et riziculture}

\section{L'intégration ancienne dans un commerce international}

Dès les premiers siècles de notre ère des sources écrites montrent que les Chinois font venir des produits originaires des forêts du nord de Sumatra, en particulier du camphre, du benjoin ${ }^{1}$ et de la résine de pin ${ }^{2}$.

Les Indiens, eux, semblent s'intéresser au camphre de cette région depuis au moins le IVe siècle, l'importation de grandes quantités ayant lieu à partir du début du $\mathrm{VI}^{\mathrm{e}}$ siècle $^{3}$, période à laquelle il est aussi connu des Grecs et des Arabes ${ }^{4}$.

L'activité commerciale ancienne de la côte est de Sumatra Nord a été confirmée localement par la fouille de Kota $\mathrm{Cina}^{5}$, un comptoir situé entre la ville actuelle de Medan et son port Belawan.

Ce site a en fait livré plusieurs milliers de pièces de monnaies chinoises ${ }^{6}$, la plupart $s^{\prime}$ échelonnant entre la seconde moitié du $\mathrm{X}^{\mathrm{e}}$ siècle et le premier quart du XII ${ }^{\mathrm{e}}$ siècle, ainsi que quelques pièces originaires de Sri Lanka ${ }^{7}$. Elles témoignent de l'intensité des échanges commerciaux internationaux. La fouille de Kota Cina a également révélé la présence de céramiques étrangères : chinoises (dont certaines de haute qualité) s'échelonnant de la seconde moitié du XII ${ }^{e}$ siècle à la deuxième partie du XIVe siècle, thaï (Sawankhalok XIve siècle) et peut-être du Moyen-Orient ${ }^{8}$.

Si les traces de marchandises importées sont par conséquent nombreuses sur le site, les données concernant les produits d'exportation sont en revanche beaucoup plus ténues. Mis à part vingt litres de résines ${ }^{9}$, il n'en subsiste aucune trace.

Des prospections menées sur la côte nord-est de Sumatra ont permis la découverte d'autres sites côtiers moins importants et plus ou moins contemporains de Kota Cina qui sont aussi liés à un commerce international ${ }^{10}$. Kota Cina apparaît ainsi, dans l'état actuel des connaissances archéologiques, comme la tête de pont d'un ensemble de petits ports de la côte nord-est de Sumatra entre le XIe et le XIVe siècle.

Ces échelles ont pu constituer l'entité politique qui apparaît pour la première fois dans les sources écrites sous le toponyme «Aru» en $1282^{11}$. Nous le retrouvons au

1. Vers 500 E.C., il semble que, en Chine méridionale, le benjoin d'Indonésie occidentale ne soit pas seulement considéré comme un substitut de la myrrhe mais commence à la supplanter grâce à de meilleures vertus fumigatoires (Wolters, Early Indonesian Commerce. A Study of the Origins of Srivijaya, Ithaca, Cornell University Press, 1967 : 111).

2. Cette résine a probablement été employée par des médecins chinois comme alternative à l'encens au VI ${ }^{\mathrm{e}}$ siècle (Wolters, $1967:$ 106-107).

3. Wolters, $1967: 68$.

4. Wolters, $1967: 149$.

5. Ce qui ne signifie nullement qu'elles sont arrivées là au vile siècle.

6. La quantité de monnaies chinoises de cette période est la plus importante jamais trouvée sur un site indonésien (Miksic, Archaeology, Trade and Society in Northeast Sumatra, PhD, Cornell university, Ithaca, $1979: 211,213$ )

7. McKinnon, Kota Cina : Its Context and Meaning in the Trade of Southeast Asia in the Twelfth to Fourteenth Centuries, $\mathrm{PhD}$, Cornell University, $1984: 110$.

8. McKinnon, $1984: 287,289$.

9. McKinnon, $1984: 119$.

10. Cf. notamment McKinnon et Luckman Sinar, « A Note on Pulau Kompei in Aru Bay, Northeastern Sumatra », Indonesia, 26, 1978 : 49-73 ; Milner, McKinnon et Luckman Sinar, "A Note on Aru and Kota Cina », Indonesia, 26, $1978: 1-42$; Miksic, 1979 : 221-244.

11. Milner, Sinar et McKinnon, $1978: 30$, 39. Pour une discussion sur la localisation d'Aru, cf. notamment Cortesao (éd. et trad.), The Suma Oriental of Tomé Pires, Londres, Hakluyt Society, Series II, vol. 89 et 90, 1944 : note 1 p. 147 ; Milner, Sinar et McKinnon, 1978 : 4-7 ; Miksic, 1979 : 47-48. 
siècle suivant parmi une liste de « régions tributaires » du royaume javanais de Mojopahit dans le Nagarakertagama.

Le Ying-yai Cheng-lan, décrivant une expédition maritime chinoise de 1413, signale «A-lu » comme producteur d'encens ${ }^{12}$. Vingt-trois ans plus tard, une autre source chinoise, le Hsing ch'a shêng lan, indique qu' «A-lu » dispose de riz, de camphre et d'autres produits aromatiques collectés dans les montagnes, et les Chinois viennent y vendre des satins et soies de couleur, des porcelaines et des perles ${ }^{13}$.

L'importance du comptoir est signalée dans la Sejarah Melayu qui fait d'Aru, à l'époque de Sultan Mansur Shah (qui règne de la fin du XIVe siècle au milieu du $\mathrm{XV}$ e siècle), une cité équivalente à Malaka ${ }^{14}$.

Quelques décennies plus tard, au début du XVIe siècle, Aru semble éclipsé par d'autres ports situés à la pointe nord de l'île. Dans ses traités de navigation, dont l'un est daté de 1511, Sulaiman b. Ahmad al-Mahri qualifie en effet le port d'Aru de petit port en comparaison de Pedir et Sumatra (Samudra) ${ }^{15}$. Il faut probablement lier cette stagnation et peut-être ce déclin au fait qu'Aru ne participe pas à la « fièvre de la culture du poivrier » qui touche le nord de l'île au moins depuis le début du XVe siècle.

Le fait est confirmé par Pirès, qui, dans sa Suma Oriental, fournit une description précise de la région vers 1520 . Trois lieux semblent à l'époque concentrer l'activité de la côte nord-est avec des commerçants étrangers : Bata (au sud de Pasai), dont la marchandise principale est le rotin ; Aru, qui possède du camphre en bonne quantité et beaucoup de benjoin, Arcat ${ }^{16}$.

Selon des légendes karo et Simalungun le royaume d'Aru se situe au bord de la rivière Wampu. D'après la famille du sultan de Deli il est situé à Deli Tua. Dans l'ancien territoire de Batubara, au sud de Serdang, la rivière Padang portait autrefois le nom de rivière Arau ou Harau (cf. Husny, Lintasan sejarah perabadan dan budaya penduduk Melayu Pesisir Deli Sumatra Timur (1612-1950), Jakarta : Dep. Pend. dan Keb., 1978 : 31). Des témoignages chinois de la dynastie Ming ainsi qu'une légende de Padang Lawas le localisent à l'estuaire ou le long de la rivière Barumun (cf. Meuraxa, Sejarah Kebudayaan Suku-Suku di Sumatra Utara, Medan, Sasterawan, 1973 : 89 ; Simanjuntak, Sejarah Batak, Balige, K. Sianapar, 1977 : 240). Dans son traité de navigation daté de 1462, Shihab Al- Din Ahmad Ibn Majid situe Aruh entre l'embouchure de la rivière Rokan et l'embouchure de la rivière Deli (cf. Tibbetts, A study of Arabic Texts Containing Material on Southeast Asia, Leiden/ London, E.J. Brill, 1979 : 198, 208). Dans ses traités de navigation datés du début du XVIe siècle, Sulaiman b. Ahmad al-Mahri localise le port d'Aruh soit à l'embouchure de la rivière Rokan, soit à l'embouchure de la rivière Barumun (Tibbetts, 1979:218,221). Sur une série de cartes de Sumatra conservées à la Bibliothèque nationale et couvrant la période 1652-1752, la Terre d'Aru (puis le royaume d'Aru) est figurée jusqu'au début du XVII ${ }^{e}$ siècle approximativement à l'embouchure de la rivière Barumun, en face des îles d'Aru (cf. cote [Ge. AF pf 195 (4919), Presqu-Isle de l'Inde au delà du Gange divisée en plusieurs peuples ou régions selon les anciens, par Sr. Sanson d'Abbeville, 1652 ; cote [Ge. D. 13231, Carte du royaume de Siam et des pays circonvoisins par le R.P Placide, 1686; cote [Ge. CC 1333, Nieuwe Kaart van het Eyland Sumatra verbeterd door François Valentyn, J. van Braam en G. Onder de Linden, in Atlas universel: l'Asie, $2^{\mathrm{e}}$ partie, t. XIX - début XVIII' siècle ?). A Panai, situé à l'embouchure de la rivière Barumun, on signale une suku Aru (cf. Schadee, Geschiedenis van Sumatra's Oostkust, Amsterdam, van Mantgem, 1919, T.1 :2-3) de même que dans le territoire voisin de Bila ou existe une suku Rau (cf. Vigelius, "Memorie van overgave van het Bestuur over de afdeeling Panei en Bila », TBG, XVII, $1869: 434$ ).

12. Mills, Ma Huan, Ying-yai Sheng-lan, The Overall Survey of the Ocean's Shore (1433), Cambridge, Hakluyt Soc. Extra series 42, Cambridge Univ. Press, 1970 : 115.

13. Rockhill, « Notes on the Relations and Trade of China with the Eastern Archipelago and the Coasts of the Indian Ocean during the fourteenth century », TP, XVI, 1915: 142.

14. Brown, Sejarah Melayu or «Malay Annals * : a Translation of Raffles Ms 18 (in the Library of the R.A.S. London) with Commentary, Kuala Lumpur, Oxford University Press, 1983 [ $1^{\text {re }}$ éd ; 1970, $1{ }^{\text {re }}$ publication dans JMBRAS 25, 2-3, 1952] : 90.

15. Tibbetts, $1979: 223$.

16. Cortesao (éd.) et (trad.), $1944: 148$. 
Nos sources sont ensuite pratiquement muettes pendant un siècle. L'émergence de la place commerciale de Deli ainsi que la prise par Aceh de celle-ci en 1612 et d'Aru l'année suivante sont parmi les rares événements touchant la région dont nous soyons certains pour cette période. Il est possible que l'important prélèvement humain effectué par Aceh après la conquête ${ }^{17}$ ait eu pour effet d'affaiblir les capacités économiques de Deli et par conséquent de tenir les marchands étrangers éloignés pour un temps.

Il faut attendre en fait la prise de Malaka par les Hollandais, en 1641, pour obtenir de nouveau des informations sur les produits disponibles à Sumatra nord-est. Les daghregister nous apprennent alors que, au milieu du XVIIe siècle, Deli, où le riz abonde, approvisionne Malaka. Les Hollandais viennent également y chercher de la cire, du bois d'aigle, des esclaves et des chevaux ${ }^{18}$.

Sur une carte de $1686^{19}$, la place d'Aru, figurée à l'embouchure de la rivière Barumun, apparaît comme importante, alors que la place de Deli (à la hauteur de l'actuelle Medan) semble moins conséquente. On sait d'autre part que, à la fin de ce même siècle, un Chinois va y négocier, dix années durant, des bassins en cuivre, des cotonnades et surtout du sel, contre du benjoin et de la cire.

Au début du XVIüe siècle, la côte nord-est apparaît fermée aux marchands étrangers ${ }^{20}$. C'est sans doute pourquoi la région est rarement mentionnée dans les sources de cette époque ${ }^{21}$. Une carte datée probablement du début du XVIII ${ }^{\text {siècle }}{ }^{22}$ mentionne la place de Deli comme la plus importante de la côte nord-est alors que le royaume d'Aru y est toujours indiqué, mais sans place de quelque puissance. Sur une carte de $1791^{23}$, aucune place conséquente n'est mentionnée sur la côte nord-est.

\section{L'organisation des échanges entre la côte et l'arrière-pays}

Cet attrait précoce des étrangers pour les produits du nord de Sumatra n'est certainement pas sans avoir influencé l'organisation politique et socio-économique de l'intérieur de l'île. Dans la partie septentrionale de Sumatra, zones de récoltes ou de productions et zones d'échanges internationaux sont généralement distinctes. L'existence d'un commerce international prospère implique alors la stabilité des relations entre région d'origine et région distributrice, afin de s'assurer la fidélité des marchands.

En consultant la liste des produits disponibles sur la côte nord-est (Bata, Aru) au début du XVI ${ }^{e}$ siècle, on constate en effet que la bande côtière n'a pas de produits de grande valeur à offrir, les plus importants, le camphre, le benjoin, l'or, le rotin et les esclaves, proviennent tous de l'arrière-pays. Ainsi, le camphrier et l'arbre à benjoin le plus recherché poussent dans le nord-ouest de l'île au-dessus de trois cents mètres d'altitude.

La présence dans l'arrière-pays de témoignages archéologiques similaires à ceux de Kota Cina permet de suggérer l'existence, à une époque ancienne, de relations étroites

17. Beaulieu parle en effet de la déportation vers Aceh de 22000 personnes originaires des royaumes de Johor, Deli, Pahang, Kedah et Perak (Beaulieu, « Voyage aux Indes orientales », in Thévenot (éd.), Collections de voyages, t. II, Paris, $1666: 114)$.

18. Daghregister 1641-1642:176, 179. Daghregister 1644-1645:68,76, 125. Daghregister $1653: 18$.

19. Bib. Nat. cote [Ge. D. 13231.

20. Hamilton, A New Account of the East Indies, Edinburgh, 1727, T.2 : 126.

21. Milner attribue lui cette rareté à la faible importance économique de la région à cette époque (Milner, Kerajaan, Malay Political Culture on the Eve of Colonial Rule, Tucson, The University of Arizona Press, 1982 : note 38 p. 149).

22. Bib. Nat., cote [Ge CC 1333.

23. Bib. Nat., cote [Ge.A.F. pf 4(43), Carte des Isles de la Sonde et du Detroit de Malaca dressée sur les mémoires de Surville, Capitaine des vaisseaux de la Compagnie, M. de Laborde, 1791. 
entre les comptoirs internationaux de la côte et les populations de l'intérieur de la région, qui semblent ainsi être intégrées dans le système de commerce à longue distance ${ }^{24}$.

Kota Cina pourrait avoir acquis son importance en raison de sa situation stratégique pour l'exploitation des portages de camphre et de benjoin en provenance des montagnes Bukit Barisan, à l'ouest et au nord du lac Toba, ainsi que de sa liaison avec le réseau de sentiers conduisant aux régions riches en or de Minangkabau et du haut Jambi. Les esclaves devaient provenir, quant à eux, du voisinage du lac Toba.

Les informations sur ces réseaux d'échanges sont rares jusqu'au début du XIX $\mathrm{X}^{\mathrm{e}}$ siècle. En effet, les dirigeants côtiers ont toujours empêché les marchands étrangers de pénétrer dans l'arrière-pays, où se trouvaient l'essentiel des ressources commercialisables.

Pirès semble être le premier à y faire allusion, mentionnant l'existence de relations commerciales par l'intérieur entre Aru et Barus ${ }^{25}$.

On sait qu'au début du XIX ${ }^{\mathrm{e}}$ siècle le trafic intérieur est généralement très important et que des habitants du piémont (dusun) et du plateau viennent régulièrement faire du commerce dans les basses terres ${ }^{26}$. Il est même des gens d'Alas, dans l'arrière-pays de Singkel, au nord de Barus, qui viennent, en passant par les montagnes, apporter camphre et benjoin. Dans l'autre sens, des marchands des basses terres vont colporter leurs produits jusqu'à six ou sept jours de marche dans l'intérieur ${ }^{27}$.

Quant au riz, nous avons vu que la côte nord-est en exporte au moins pendant la période $\mathrm{XV}^{\mathrm{e}}$-XVIIe siècle.

Deli, qui prend le relais d'Aru au XVIre siècle, semble d'abord mettre l'accent sur le développement de la production rizicole, basé sur l'importation de ferraille permettant la confection d'outils performants pour le défrichement ${ }^{28}$.

Cette production a pu susciter l'intérêt des comptoirs poivriers du nord. Nous savons en effet que tout au long de son histoire, le sultanat d'Aceh fut confronté au problème de l'approvisionnement en riz pour nourrir sa population ${ }^{29}$. Or, dès le début du $\mathrm{XVI}^{\mathrm{e}}$ siècle, le plateau au nord du lac Toba ainsi que la plaine côtière pouvaient faire office de « grenier à riz ${ }^{30}$.

Une tradition de l'ouest du plateau pourrait constituer un premier élément pour confirmer cette hypothèse. En effet, à la fin du XIX siècle, on croit encore à Kuala que les récoltes de riz ne réussissent qu'aussi longtemps que les habitants accordent le pas-

24. Des céramiques d'importation anciennes (sans doute des assiettes céladon) utilisées comme mobilier funéraire ont ainsi été retrouvées vers 1930 dans les montagnes du sud de l'île de Samosir sur le lac Toba (Schnitger, Forgotten Kingdoms in Sumatra, Singapore, Oxford University Press, 1989 [1 1 re éd. 1939] : 107-108). D'autre part, des céramiques importées du XIve siècle ont été recueillies à Deli Tua sur un site surplombant la rivière Deli à quelque trente kilomètres au sud de Belawan ainsi qu'à Siberaya au bord de la rivière Wampu sur le plateau au nord du lac Toba, non loin de Kabanjahe (Miksic, 1979 : 230-237). Dans la même région, à Lingga, nous avons pu voir une jarre yüan (XIII ${ }^{{ }_{-}}$ XIVe siècle) (nous remercions Mme M.F. Dupoizat pour son identification) conservée dans un petit musée privé.

25. Cortesao (éd.) et (trad.), $1944: 148$.

26. Anderson, Mission to the East Coast of Sumatra in 1823, Edinburgh, W. Blackwood/London, T. Cadell Strand, 1826 : 263 ; De Scheemaker, « Aanteekeningen, gehouden op eene reis naar de marktplaats (Pedagangan) der Lima Laras, vier dagreizen de rivier van Batoe Bara opwaarts gelegen ", $T B G$, XVII, $1869 \mathrm{~b}: 412$.

27. Anderson, $1826: 263$.

28. Cf. par exemple daghregister $1682(2): 929$.

29. Lombard, Le sultanat d'Atjeh au temps d'Iskandar Muda (1607-1636), PEFEO 61, Paris, $1967: 60-61$.

30. Pirès observe à propos d'Aru : « The land of Aru has plenty of rice, and very white and good and in large quantities [...] ». Il indique également que Bata produit du riz (Cortesao [éd.] et [trad.], $1944: 146,148$ ). 
sage aux Acihais. D'autre part, à la même époque, le blocus côtier imposé par les autorités coloniales pendant la guerre d'Aceh entraîne l'intensification des incursions sur le plateau de groupes venus du sultanat pour s'approvisionner en riz. Il ne s'agit peut-être là alors que de la renaissance d'un ancien réseau d'échanges. Par ailleurs, la réactivation, à une époque indéterminée, de l'institution des «quatre Raja » (Raja Berempat) sur le plateau au nord du lac Toba par le grand sultanat du nord pourrait s'expliquer comme un moyen d'obtenir des approvisionnements réguliers en riz en s'assurant la fidélité de hauts personnages par l'octroi d'un statut.

La toponymie atteste l'existence, dans cette zone de transition qu'est le piémont, de lieux d'échanges et de rencontres. Deux toponymes reviennent à de nombreuses reprises. Le plus fréquent est composé avec «bandar», défini localement comme "l'endroit où les gens des basses terres viennent faire du commerce avec les gens des hautes terres " ${ }^{31}$. Nous n'en avons pas relevé moins de trente-sept entre le cours inférieur de la rivière Wampu et le cours supérieur de la rivière Bulian (carte p. 159). L'autre toponyme, «Pertumbukan », qui signifie « rencontre », apparaît à cinq reprises.

La plupart de ces toponymes sont concentrés dans une bande d'une trentaine de kilomètres de largeur dont l'altitude varie de cinquante mètres à environ mille mètres. $\mathrm{A}$ l'intérieur de cette bande, trois zones sont repérables : deux abondent en toponymes composés avec «bandar », il s'agit du cours supérieur de la rivière Binjei et de ses affluents, ainsi que du cours supérieur des rivières Tuntungan et Klumat, mais c'est encore plus marquant dans la zone comprenant le cours supérieur de la rivière Batugingging et ses affluents ainsi que le cours supérieur de la rivière Ular et de ses affluents. Entre ces deux zones, à savoir les vallées des rivières Deli, Percut et Belumai, ce toponyme est plus rare.

En règle générale, ces toponymes sont situés le long des rivières. Si, en effet, la circulation sur le plateau au nord du lac Toba ne peut se faire autrement que sur des sentiers, dans le piémont, par contre, deux solutions sont possibles : le sentier ou la voie d'eau. Plusieurs points de passages permettent de franchir les montagnes pour atteindre les basses terres de Deli, Serdang et l'est de Langkat : Buaya, Liang, Negeri, Cingkem, Betimus, Serbanyaman et Bekancan ${ }^{32}$, les plus utilisés étant Buaya et Cingkem. On sait qu'il existait des sentiers servant de voies commerciales où le cheval était utilisable entre le plateau ${ }^{33}$ et les basses terres de Serdang, Deli et Langkat ${ }^{34}$. Il y avait aussi des chemins permettant de relier différentes vallées, tel celui entre Deli Tua sur la rivière Deli, Petumbah sur la rivière Percut et Tatukanraga sur la rivière Belumai ou encore entre la rivière Mencirim et la rivière Wampu.

Les données sont moins sûres en ce qui concerne les portions navigables des rivières. D'une part, certains témoins, considérant la rivière navigable à partir du moment où des embarcations d'une certaine envergure peuvent y circuler, négligent le trafic réalisé par des esquifs ; d'autre part, l'alluvionnement est si rapide que les conditions de navigation peuvent se modifier en quelques décennies. Les quelques informations à notre disposition émanent des premiers voyageurs ayant pénétré dans l'intérieur et des riverains (carte p. 159).

Si l'on s'en tient à ces données, la localisation des bandar n'aurait aucune relation avec la limite de navigabilité des cours d'eau. Nos informations sur ces conditions sont

31. Kroesen, « Geschiedenis van Asahan », TBG, XXXI, $1886: 90$.

32. Westenberg, « Nota over de onafhankelijke Bataklanden », TBG, XXXIV, $1891: 106$; Engelbert van Bevervoorde, « Een bezoek aan de bataksche hoogvlakte », BKI, XLI, 1892 : 611-612 ; Wijngaarden, « Verslag omtrent de zending onder de Karau-Bataks over 1893 », MNZ, 38, 1894 : 137 ; Volz, Nord-Sumatra. Bd. 1 : die Batakländer., Berlin, Dietrich Reimer, 1909, T.1 : $232-233$; Joustra, Batakspiegel, Uitgave van het Bataksch Instituut no 3, Leiden, S.C. van Doesburgh, $1910: 51$.

33. Notamment à partir de Tongging sur la rive nord du lac Toba.

34. De Haan, « Verslag van eene Reis in de Bataklanden », VBG, 38(2), 1875 : 7. 


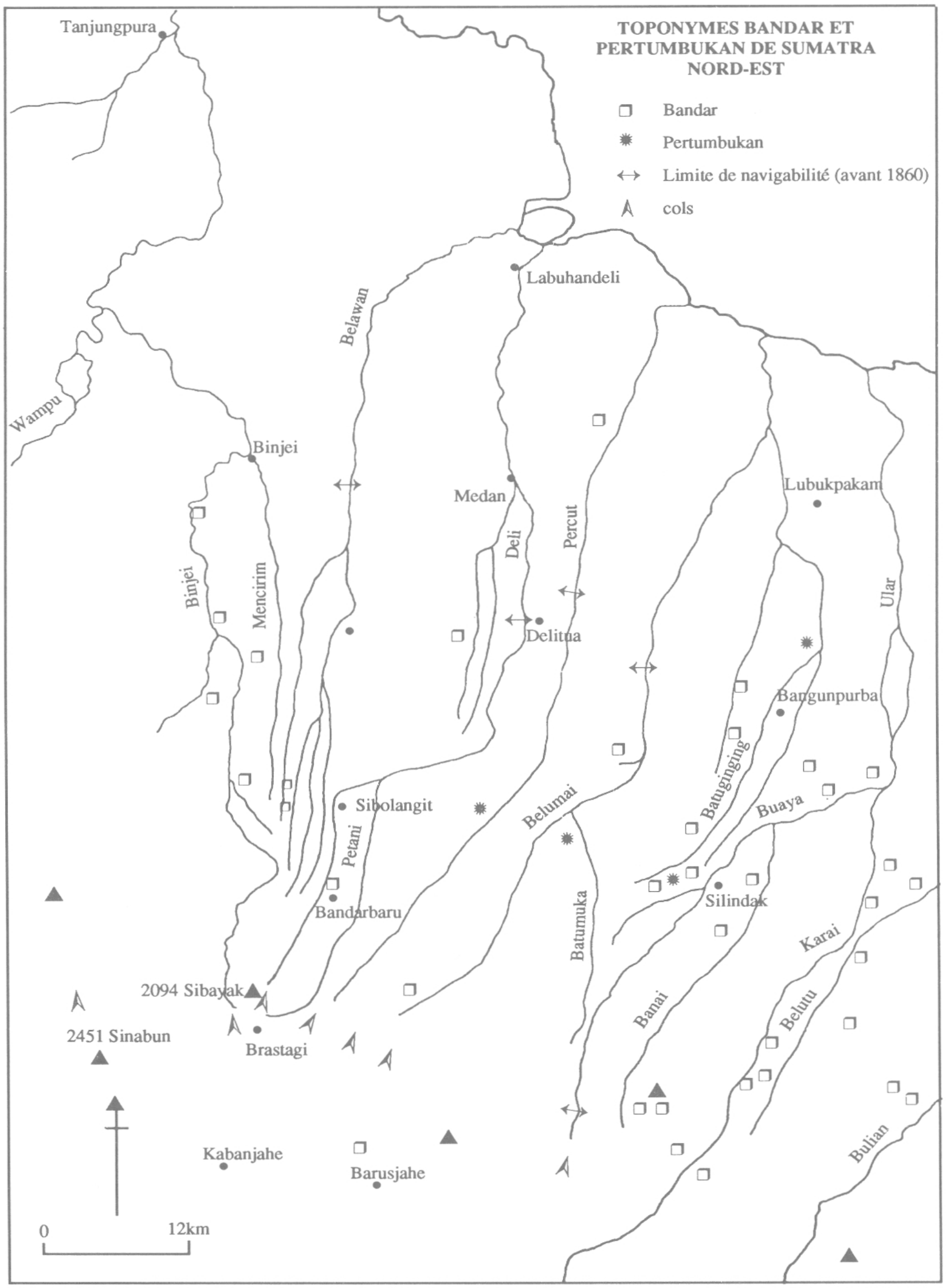


toutefois trop fragmentaires pour le prouver de manière indubitable. Il est également impossible pour l'instant de connaître l'ancienneté de ces toponymes ainsi que leur éventuelle contemporanéité. Cependant, plusieurs d'entre eux devaient encore être en vigueur à l'arrivée des Néerlandais. Enfin, leur rôle véritable (péage, octroi ou simple débarcadère...), ainsi que les groupes sociaux qui en avaient le contrôle, reste encore à déterminer.

Des rapprochements avec des données postérieures incitent à penser que les interactions entre les gens de la côte et ceux de l'arrière-pays devaient être réglées par des serments et des accords ${ }^{35}$. On sait ainsi, grâce à des inscriptions gravées sur des assiettes en cuivre ou en argent, qu'au XVIIre siècle, dans la région de Palembang, certains produits disponibles dans les hautes terres sont le monopole de dirigeants de Palembang et doivent être fournis selon des règles spécifiques ${ }^{36}$.

Il est possible, dans cet ordre d'idée, que les toponymes côtiers actuels « Belawan » (à l'embouchure de la rivière Belawan) et « Bedagai », qui signifient en langue karo «faire un accord par serment » soient les traces d'une époque où les populations de l'intérieur venaient promettre de fournir tel ou tel produit à des marchands étrangers ${ }^{37}$.

En contrepartie de la fourniture des produits de cueillette et du riz, les côtiers réglaient leur transaction non seulement en sel et autres produits de première nécessité, mais aussi en monnaies. L'insertion de l'arrière-pays dans un espace monétaire « sinisé » approvisionné par des comptoirs tels que Kota Cina reste pour l'instant au stade de l'hypothèse. En revanche, nous savons qu'à partir du XVIe siècle la côte nord-est et son arrière-pays sont intégrés dans une zone monétaire régionale basée sur des monnaies acihaises ${ }^{38}$ et, à partir de la deuxième moitié du XVIII ${ }^{e}$ siècle, dans une zone monétaire internationale basée sur des dollars espagnols. D'autre part, des céramiques importées étaient probablement offertes pour rehausser le statut et s'assurer le concours d'individus chargés de réguler l'approvisionnement des comptoirs, surtout en camphre et en benjoin.

De ces données il ressort que, au moins depuis le XII e siècle, la côte nord-est est l'aboutissement de réseaux d'échanges irriguant l'arrière-pays et parcourus par des groupes d'individus migrants temporairement.

Il est par conséquent vraisemblable que, très tôt, deux élites aient émergé : une dans l'arrière-pays ${ }^{39}$, contrôlant la cueillette et le transport des produits forestiers et du riz

35. Drakard, A Malay Frontier: Unity and Duality in a Sumatran Kingdom, Ithaca, Cornell University, Southeast Asia Program, $1990: 181$.

36. Miksic, $1979: 108$.

37. Selon une légende, il y aurait eu autrefois à Belawan un keramat sur lequel les marchands de l'arrière-pays allant vendre des chevaux à Penang faisaient le serment qu'ils ne voleraient pas leurs semblables (cf. McKinnon, 1984 : 16). Nous ferons observer d'autre part que ce toponyme apparaît également tout proche de la source de cette rivière dans le dusun.

38. A Deli Tua et dans les environs, on aurait retrouvé notamment deux guci contenant des pièces d'or. L'un d'eux, découvert en 1902 sur une plantation de Deli Tua, contenait des pièces datées du dernier quart du XVII ${ }^{\mathrm{e}}$ siècle (Meuraxa, 1973: 84). Les guru traditionnels du plateau au nord du lac Toba, qui enseignent la lecture et l'écriture de la langue vernaculaire sont encore, en 1875, rémunérés en monnaie d'or (drahan), très certainement les dirham du nord de l'île (De Haan, $1875: 16$ ). D'autre part, au début du $\mathrm{XX}^{\mathrm{e}}$ siècle, sur les deux grands marchés de Pane, une principauté de Simalungun au bord du lac Toba, la monnaie d'or acihaise est encore en vigueur (Kroesen et Helderman, "Mededeelingen betreffende het landschap Panei en het Rajahgebied », BKI, LVI, 1904 : 569). Enfin dans l'adat des populations du plateau au nord du lac Toba, des offrandes en mas sont prévues dans les transactions matrimoniales encore au début du $\mathrm{XX}^{\mathrm{e}}$ siècle (cf. Joustra, "Het leven, de zeden en gewoonten der Bataks », MNZ, 46, 1902a : 399).

39. Ce sont peut-être leurs descendants qui, en s'appropriant le commerce des chevaux (dont la stimulation est sans doute à mettre en relation avec l'expansion des mines d'étain en péninsule malaise), des esclaves et de l'opium vont renforcer la position dominante de certaines lignées de l'arrière-pays. 
vers la côte ; une sur la côte, basant sa richesse sur le monopole des échanges avec l'extérieur.

\section{Les plantations autochtones précoloniales}

Lorsque John Anderson, un Anglais au service de l'East India Company, prospecte la côte nord-est de Sumatra en 1823, il constate qu'une flotte locale dépassant certainement les mille embarcations ${ }^{40}$ transporte vers la péninsule malaise non seulement des produits forestiers tels que benjoin, camphre, résines, rotin, cire, bois de teinture, mais également des produits agricoles tels que riz, légumes, gommes, tabac, gambier, et surtout du poivre ${ }^{41}$.

Anderson est en fait le premier Européen témoin direct de l'existence de ces plantations à Deli.

Leur développement introduit un bouleversement du système de relations liant la côte à l'arrière-pays. De simple intermédiaire dans la négociation de produits de cueillette, la côte nord-est devient elle-même productrice, mobilisant à la fois un savoir-faire nouveau, des terres, des moyens techniques et une main-d'œuvre importante.

\section{La question des origines}

Nous ne disposons pas d'éléments permettant de dater avec précision l'apparition des plantations de poivriers sur la côte nord-est de Sumatra ${ }^{42}$. Alors que ni Pirès ni Mendés Pinto n'indiquent le poivre comme une production de cette région dans la première moitié du XVI ${ }^{e}$ siècle, un routier portugais de la fin du XVIe siècle signale que le poivre vendu à Aceh provient pour partie des montagnes d'Aru ${ }^{43}$. Aucun daghregister ne semble mentionner la culture du poivrier sur la côte est de Sumatra-Nord jusqu'en 1682. Tout ce qu'en disent les fonctionnaires néerlandais à leur arrivée dans les années 1860, c'est que les tribus «batak » du nord de Sumatra ont depuis de nombreuses années planté presque exclusivement du poivrier ${ }^{44}$.

La première mention certaine remonte à la fin du XVIII ${ }^{e}$ siècle dans un rapport britannique indiquant que Langkat, frontalier avec Aceh, produit du poivre ${ }^{45}$. Un témoi-

40. Six cents pour la seule région de Batubara (d'après le syahbandar du lieu) au sud de Deli, dont les marins sont les principaux transporteurs internationaux de la région (Anderson, $1826: 312$; Anderson, Acheen and the Ports on the North and East Coast of Sumatra, London, 1840 : 194). Leurs embarcations contiennent de huit à dix-huit kojang (soit de dix à vingt-quatre tonnes) (De Scheemaker, « Nota betreffende het landschap Batoebarah », TBG, XVII, 1869a : 475). Langkat possède environ deux cents embarcations allant de deux à trente tonnes (dont huit au moins de ce dernier type) (Anderson, $1826: 250$ ). Le même auteur signale que Serdang possède un grand nombre d'embarcations qui convoient les produits, entre autres vers Penang (Anderson, $1826: 303$ ).

41. Deli produit alors annuellement 26000 pikul, soit 1300 tonnes, Langkat exportant quant à lui 20000 pikul (Anderson, 1826:246, 260 ; Veth, « Het landschap Deli », TNAG, II, $1877: 158$ ).

42. Plus au sud sur la côte est, on sait qu'en 1823 le poivrier vient d'être essayé dans l'arrièrepays d'Asahan (cf. Anderson, $1826: 128,322$ ). Sur la côte ouest, Singkel fournit déjà du poivre au début du XVI siècle (cf. Cortesao [éd.] et [trad.], $1944: 163$ ) alors que Natal commençe à en produire seulement à partir de 1810 et en exporte 500000 livres en 1823 (cf. Dobbin, Islamic Revivalism in a Changing Peasant Economy : Central Sumatra, 1784-1847, Copenhagen, 1983 : 172).

43. Dos Santos Alves, « Une Ville inquiète et un Sultan barricadé : Aceh vers 1588 d'après le Roteiro das Cousas do Achem de l'Evêque de Malaka », Archipel, 39, $1990: 105$.

44. Netscher, « Togtes in het gebied van Riouw en Onderhoorigheden », TBG, 14, $1864: 345$.

45. Wurtzburg, «A Brief Account of the Several Countries Surrounding Prince of Wale's Island with their Production. Recd. from Captain Leight in 1789 », JMBRAS, XVI, Part I, 1938 : 123-126. 
gnage recueilli par un fonctionnaire néerlandais indique qu'à la même époque deux personnages originaires de Siak auraient été chassés et auraient émigré vers Langkat, où ils se seraient enrichis grâce au commerce du poivre ${ }^{46}$.

Qui est à l'origine de cette innovation ? Peut-être un pionnier venu du nord de l'île, à l'image du Néerlandais Jacob Nienhuys, qui, en 1863, viendra de Java y développer avec succès la culture du tabac ou alors un autochtone qui, après un séjour à Aceh, est revenu tenter l'aventure ? Aucune tradition locale ne mentionne explicitement la présence à Deli de planteurs acihais. Il est d'ailleurs peu probable que l'installation des plantations de poivriers à Deli, si elle a lieu au cours du XVIII e siècle, résulte d'une volonté politique du sultanat d'Aceh. Ce dernier se débat en effet à l'époque dans de grandes difficultés intérieures.

Certaines sources tendent plutôt à montrer que des non-Acihais auraient joué, peutêtre par l'intermédiaire d'Aceh, un rôle non négligeable dans ce développement.

Ainsi, les généalogies et les traditions locales présentent quatre personnages autochtones à Sumatra-Nord, sinon comme les promoteurs, du moins comme des entrepreneurs dynamiques qui vont développer ces plantations. L'examen de leur itinéraire (carte p. 163) montre que tous résident sur le plateau au nord du lac Toba avant de se rendre vers le piémont et les basses terres et que deux d'entre eux séjournent à Aceh.

Le premier, Si Sembiring Meliala, est descendant de Tamouls et originaire du plateau au nord du lac Toba. Cadet d'une famille de deux enfants, il quitte le plateau pour se diriger vers le piémont en suivant la rivière Deli. Il s'installe dans une cabane au bord de la rivière, épouse une fille du lieu, défriche et cultive du riz et du poivre, qu'il vend sur la côte. La population s'accroissant aux alentours, il fonde alors Deli Tua un peu plus en aval. Son frère aîné meurt pendant ce temps, laissant une épouse enceinte qui mettra au monde la fameuse Puteri Hijau et ses deux frères. Puteri Hijau se marie avec un haut personnage du plateau, qu'elle est bientôt contrainte de quitter, et s'en va avec ses deux frères s'installer à Deli Tua, fondé par leur oncle, et dont elle va étendre le territoire ${ }^{47}$. Au vu des premières prospections archéologiques menées à Deli Tua, le site semble avoir été habité au moins dès le XIVe siècle, ce qui ferait remonter l'existence de la culture du poivrier à cette époque si l'on s'en réfère à la tradition ${ }^{48}$.

La seconde figure est Guru Patimpus. Pour les uns, petit-fils du réputé « roi-prêtre » installé sur la rive sud du lac Toba à Bakkara, Si Singamangaradja ${ }^{49}$, pour les autres, originaire du plateau au nord du lac Toba, il est l'un des personnages principaux de la Riwayat Hamperan Perak. Après avoir ramené la paix sur le plateau et y avoir pris des épouses, il s'en va à Langkat chercher des terres fertiles pour y cultiver le poivrier dans un but commercial. Il y fonde un village, puis revient sur le plateau, où il entend alors parler d'un personnage exceptionnel, un Arabe musulman venant de Java, installé sur le territoire de Deli. Guru Patimpus décide de le rencontrer et descend dans les basses terres en établissant de nombreuses colonies le long de son itinéraire. Il se convertit à l'islam, épouse la fille d'une autorité locale et fonde Medan. Le nombre des immigrants venus du plateau augmente ainsi que le nombre de plantations de poivriers ${ }^{50}$.

46. Mendelaar, Mvo Padang en Bedagai, 1930 : 12-13.

47. Brahma Putro, Karo dari Jaman ke Jaman. Jil. 1., Medan, Yayasan Massa, 1981 : 214-223.

48. Le site a pu aussi avoir été abandonné puis réoccupé plus tard.

49. Les descendants de Si Singamangaradja XII, en particulier Raja Barita Sinambela contestent cette filiation (Husny, $1978: 52$ ).

50. DPRD Kotamadya Daerah Tingkat II Medan, Dokumenta Pengabdian D.P.R.D. Kotamadya Daerah Tk. II Medan (1971-1976), Medan, Sekretariat D.P.R.D. Kotamadya Daerah Tk. II Medan, s.d. [1977 ?] : 599-603 ; Simanjuntak, 1977 : 214-217. 


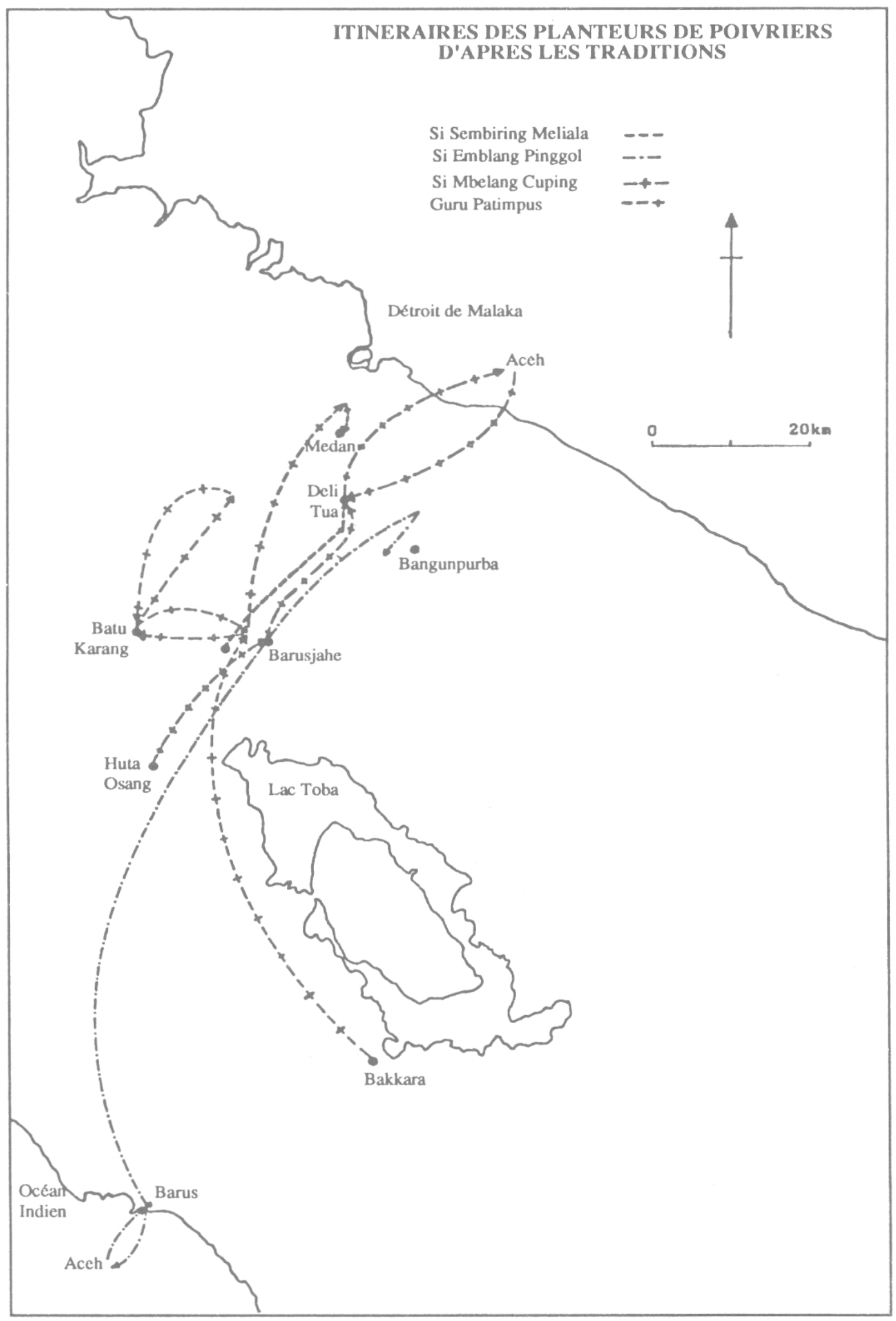


Guru Patimpus est l'ancêtre supposé des dirigeants (datuk) de la fédération de villages (urung) d'Hamperan Perak (aussi nommé XII Kuta) ${ }^{\text {s1 }}$. L'un d'eux, Sutan Amad, est encore mineur lors de la visite d'Anderson en 1823, lequel sera frappé par la bonne tenue des plantations de poivriers sur son territoire ${ }^{52}$. La généalogie en faisant un membre de la sixième génération après Guru Patimpus, l'ouverture des plantations de poivriers à Langkat remonterait alors, au plus tôt, au milieu du XVII ${ }^{\mathrm{e}}$ siècle.

Le troisième personnage, Si Emblang Pinggol, est originaire de Barus. Après un séjour à Aceh, où il épouse la fille d'un haut personnage ${ }^{53}$, il revient à Barus, traverse le plateau au nord du lac Toba et fonde, dans le piémont, Pertumbukan, un village connu pour sa richesse en plantations de poivriers dans la seconde moitié du XIXe siècle ${ }^{54}$. Si Emblang Pinggol est l'ancêtre supposé des dirigeants (kejuruan) de la fédération de Senembah. L'un d'eux, nommé kejuruan en 1862, appartient, selon la généalogie $^{55}$, à la sixième génération, ce qui renverrait la fondation de Pertumbukan à la première moitié du XVIII ${ }^{e}$ siècle.

Le dernier personnage, Si Mbelang Cuping («Grandes Oreilles ») est originaire de l'ouest du lac Toba. Après avoir fondé un village sur le plateau, il s'installe dans le piémont, au sud de Deli Tua. Devenu riche (nous pensons qu'il s'enrichit grâce à la culture du poivre), il se rend à Aceh chercher un titre et épouser une Acihaise.

Revenu à Deli, il aura, d'un nouveau mariage avec une fille de la région, un fils qui sera l'ancêtre de plusieurs dirigeants d'une fédération du plateau. Nous savons que, dans les années 1920, l'un des «nobles » (sibayak) du piémont appartient à la neuvième génération des descendants de Si Mbelang Cuping ${ }^{56}$, ce qui ferait remonter les activités de ce dernier sur la Côte Est à la première moitié du XVIII ${ }^{e}$ siècle, tout comme $\mathrm{Si}$ Emblang Pinggol.

L'origine des plantations de poivriers dans la région reste donc à élucider. Rien n'interdit toutefois de penser qu'elle pourrait être déjà un fournisseur d'Aceh dès la seconde moitié du XVIe siècle lorsque le grand sultanat prend le contrôle de la côte pour un temps.

\section{Un essai de localisation}

La localisation des plantations pose également un problème, et il faut attendre le début du XIX ${ }^{\mathrm{e}}$ siècle pour savoir qu'il en existe à quelques kilomètres seulement de la côte ${ }^{57}$. Les rapports des premiers fonctionnaires néerlandais et les souvenirs des autochtones sont également utiles. Mais, là encore, il faut faire appel à la toponymie pour tenter de compléter la carte (carte p. 167). La présence du terme lada nous paraît tout à fait logique dans la mesure où il est utilisé en malais, en «batak » et en acihais ${ }^{58}$.

51. Il y a près de Hamperan Perak, à Kampung Lama, un keramat Guru Patimpus. Medan possède également son keramat Guru Patimpus.

52. Anderson, $1826: 61,256,259$. La Riwayat Hamperan Perak mentionne que c'est le grandpère de Sutan Amad, Ali, qui a ouvert ces plantations de poivriers (DPRD, $1977: 603$ ).

53. Panglima Polim. Nous savons d'après les généalogies et les traditions que les Panglima Polim sont les dirigeants successifs du sagoë de 22 mukim sensé naître sous le règne de Noer al-Alam entre 1675 et 1678. Reid, "Trade and the Problem of Royal Power in Aceh. Three Stages : c. 1550-1700", in A. Reid et L. Castles (ed.), Pre-colonial State Systems in Southeast Asia, Monograph no 6 of MBRAS, Kuala Lumpur, 1975 : 53.

54. Luckman Sinar, Sari Sejarah Serdang, Jakarta, Dep. Pend. dan Kebud., 1986, T.1 : 145-146. Hagen, « Beiträge zur Kenntnis der Battareligion», TBG, 28, 1883a : 42, 43.

55. Luckman Sinar, 1986, T.1 : 147-150.

56. Neumann, « Bijdrage tot de Geschiedenis der Karo-Batakstammen », BKI, 82, 1926: 9-10.

57. Un contrôleur néerlandais affirme même qu'à une certaine époque les plantations de poivriers s'étendaient jusqu'à la côte (cf. Kok, Mvo doesoen Deli-Serdang, 1910 : 150).

58. K. Heyne, 1927, T.1: 527. 
Dans la région qui nous intéresse, nous avons relevé quatre toponymes avec lada dont deux kebun lada, qui correspondent effectivement à des plantations notées par Anderson.

$\mathrm{Ni}$ la toponymie ni les rapports des premiers fonctionnaires et missionnaires ne font état de la présence de plantations de poivriers sur le plateau au nord du lac Toba. Nous savons simplement qu'en 1823 un "noble » du plateau fournit du poivre ${ }^{59}$ et qu'il existe à Langkat des plantations à cinq ou six jours de la côte ${ }^{60}$.

Nous sommes persuadé que la carte présentée ici est très incomplète et que de nombreuses enquêtes seraient nécessaires (en remontant notamment les rives des principaux cours d'eau) pour appréhender l'importance de l'espace consacré aux plantations de poivriers dans la première moitié du XIX ${ }^{e}$ siècle.

Grâce à la toponymie nous savons d'autre part que des plantations de gambier et de cotonniers coexistaient avec les plantations de poivriers. En effet, traditionnellement, le piémont est divisé en deux zones appelées sinuan bunga et sinuan gambir, nuwan renvoyant à l'idée d' « espace cultivé », aussi bien en rizières que par d'autres types de cultures ${ }^{61}$.

L'expression sinuan bunga est appliquée à la partie basse du piémont et trouverait son origine dans le fait que cette portion du territoire était consacrée à la culture du cotonnier désigné localement bunga kembajat ${ }^{62}$.

De la même manière, l'expression sinuan gambir, appliquée à la partie haute du piémont, indiquerait à l'origine un espace consacré à la culture du gambier dont les feuilles entrent dans la composition de la chique de bétel et sont également exportées pour leurs propriétés médicinales ${ }^{63}$.

\section{L'évolution de la production}

Si nous ne disposons d'aucune statistique sur la production de poivre avant le début du XIXe siècle, une donnée est par contre certaine, c'est le grand développement de la production à partir de cette époque.

Milner pense que les ambitions commerciales du dirigeant de Deli, Sultan Amaluddin Panglima Mangedar Alam (qui semble décéder vers 1825), ont pu être encouragées par la crise politique à Aceh. En effet, pendant cette période de rareté du poivre au niveau mondial, de guerre civile à Aceh, une demande se crée à Penang pour d'autres fournisseurs. Mangedar Alam y aurait répondu en développant la culture du poivrier sur ses territoires.

La culture du poivrier sur la côte nord-est de Sumatra apparaît donc avant tout comme une culture d'exportation. Des rapports britanniques suggèrent qu'à Deli les exportations de poivre se sont au moins multipliées par dix entre 1815 et $1822^{64}$. Il faut certainement lier cette expansion à l'importation massive d'outils et de fer d'Aceh ou de péninsule malaise ${ }^{65}$, fer qui permet la fabrication d'outillage agricole facilitant les défrichements. Anderson estime que, pour cette année 1822, les quatre places de la côte

59. Anderson, $1826: 296$.

60. Anderson, $1826: 104$.

61. Joustra, Karo-Bataksch woordenboek, Leiden, E.J. Brill, $1907: 203$.

62. Neumann, Karo-Batak-Nederlands woordenboek, Medan, Varekamp \& Co., 1951 : 145.

63. Westenberg, «Verslag van eener reis naar de onafhankelijke Bataklanden ten noorden van het Tobameer », TNAG, XIV, $1897: 20$; De Ridder, De invloed van de westersche cultures op de autochtone bevolking ter Oostkust van Sumatra, Proefschrift Leiden. Wageningen, H. Veenman, 1935 : 40.

64. Milner, 1982 : 15.

65. On sait grâce à Anderson que Deli importe des outils et du fer de la péninsule malaise. D'autre part, Aceh est à cette époque également un grand importateur de fer (Lombard, Le carrefour javanais. Essai d'histoire globale, Paris, EHESS, 1990, T.1 : 111). 
nord-est que sont Langkat, Buluh Cina, Deli et Serdang ont exporté près de 3500 tonnes de poivre vers Malaka, Penang et Singapour ${ }^{66}$. Il semble que, jusqu'en 1845 le commerce du poivre stagne avec Penang. Mais il connait un boom entre 1853 et 1859 , qui se traduit par un doublement de l'activité et une valeur atteignant 1 million de dollars espagnols ${ }^{67}$.

\section{L'institution « Datuk Empat Suku » comme moteur de l'expansion des plantations}

Sur le plan intérieur, si c'est bien, comme l'indique le Hikayat Deli, Sultan Amaluddin Panglima Mangedar Alam, dont l'ancêtre serait un Indien ayant connu la gloire à Aceh, qui est l'initiateur de la réorganisation de l'arrière-pays de Deli avec la mise en place de l'institution « Datuk Empat Suku » ${ }^{68}$, peut-être alors faut-il interpréter cette initiative comme un mouvement fédérateur lancé par le sultan pour défricher la forêt environnante et permettre ainsi le développement des plantations, notamment celles de poivriers, qui se produit durant son règne.

Ainsi, la fédération de ce qui nous apparaît, au moment où nous disposons de sources suffisamment précises, comme quatre groupes territoriaux non exogames, doit peut-être sa réalisation à des stratégies politiques grâce auxquelles s'élaborent des systèmes d'alliances obligeant les membres de ces suku à remettre tout ou partie de leur production à un partenaire bien défini, en l'occurrence le sultan de Deli. Au prestige d'une origine acihaise, celui-ci aurait alors ajouté celui d'avoir réussi à multiplier les alliances pour asseoir son autorité.

On comprendrait d'autre part que, si celui -ci est d'origine étrangère, il ait eu besoin d'user de telles stratégies afin d'obtenir le soutien dans la population locale d'individus capables de mobiliser les espaces et la main-d'œuvre nécessaires à l'expansion des plantations ${ }^{69}$.

De la même manière, les traditions locales semblent nous indiquer que les «promoteurs », tous étrangers à la Côte Est, prennent des épouses parmi l'élite locale afin d'avoir accès non seulement à la terre, mais aussi à la main-d'œuvre.

Nous pensons en effet que la colonisation agricole de la côte nord-est, et de Deli en particulier, n'a pu réussir que grâce aux liens généalogiques unissant les chefs d'urung (fédération de «villages ») des basses terres (datuk et kejuruan), d'une part, et les sibayak et raja de l'est du plateau, d'autre part. En effet, du point de vue de la population, les quatre fédérations des basses terres de Deli (XII Kuta, Sukapiring, Sunggal, Senembah) sont les prolongements des quatre fédérations de l'est du plateau (XII Kuta, Sukapiring, Telu Kuru, Si Pitu Kuta). Ainsi, les chefs d'urung eux-mêmes sont des descendants d'individus originaires du plateau.

Ces rapports privilégiés entre fédérations de villages des basses terres et fédérations de villages du plateau ont certainement permis aux dirigeants de la côte de faire appel à la main-d'œuvre utile au développement des plantations.

66. Respectivement, Langkat 20000 pikul (le pikul équivaut à environ $50 \mathrm{kgs}$ ) vers Penang et Malaka ; Buluh Cina 15600 pikul vers Penang et Malaka ; Deli 26000 pikul vers Malaka, Singapour et Penang ; Serdang 8000 pikul vers Malaka et Penang (Anderson, 1826: 246, 260, 279, 304).

67. Braddell, 1861 (cité par Luckman Sinar, «The Impact of Dutch Colonialism on the Malay Coastal States on the East Coast of Sumatra during the 19th Century », in Papers of the Dutch-Indonesian Historical Conference Held at Noordwijkerhout, the Netherlands, 19 to 22 May 1976, Leiden/Jakarta, $1978: 181$ ).

68. Hikayat Deli : 195 .

69. Si Mangedar Alam bénéficie du soutien d'Aceh à l'origine, les tentatives d'Aceh pour exercer un contrôle sur le plateau avec la « réactivation » du « Radja Berempat » pourraient être contemporaines du mouvement de fédération à Deli dans le but d'assurer la stabilité d'un réservoir de main-d'œuvre. 


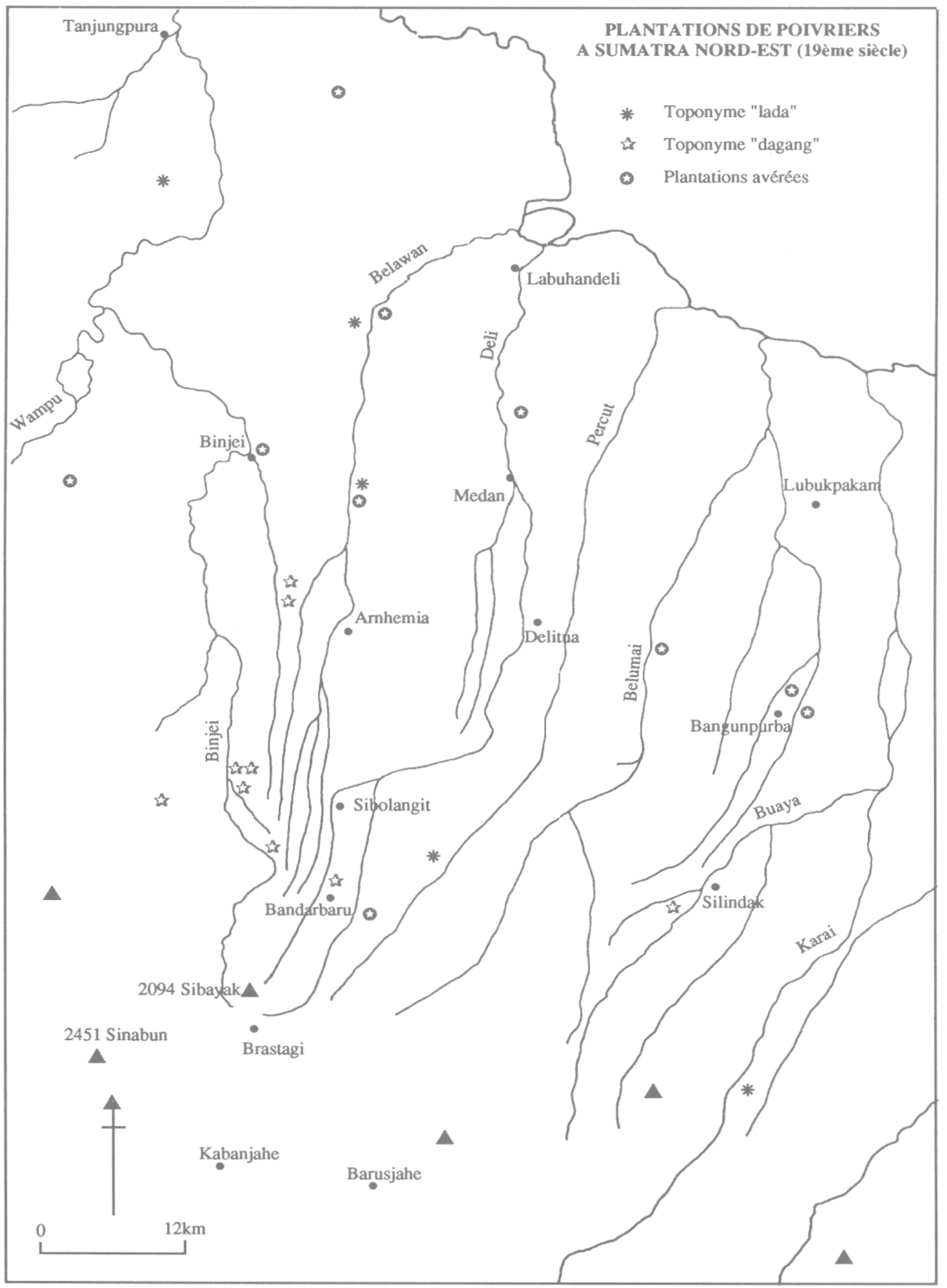




\section{L'organisation des plantations}

On imagine le bouleversement des traditions agricoles et foncières locales à l'occasion du passage d'une agriculture sur brûlis à une agriculture de plantations. Si l'organisation des plantations indigènes de poivriers est relativement bien connue pour la côte est d'Aceh ${ }^{70}$ et le nord de Langkat ${ }^{71}$, il n'en va pas de même plus au sud, notamment à Deli et Serdang. Dans les basses terres et le piémont, les dirigeants apparaissent, au début du XIX $\mathrm{X}^{\mathrm{e}}$ siècle, comme les principaux propriétaires de plantations de poivriers ${ }^{72}$. A Deli en 1823 , le syahbandar est, selon Anderson, le plus grand marchand de poivre du sultanat ${ }^{73}$.

Un rapport hollandais confirme qu'avant la soumission de Deli à l'autorité de Batavia la plupart des chefs de fédérations des basses terres et du piémont sont propriétaires de telles plantations ${ }^{74}$, et le résident Netscher observe en 1864 que les «Batak » doivent dans des conditions bien précises planter des poivriers pour le compte du sultan de Deli ${ }^{75}$.

Ces dirigeants disposent d'un personnel d'encadrement originaire de l'arrière-pays.

A côté de ceux que l'on pourrait qualifier de «grands planteurs », certains indices permettent de penser qu'il existe des petits planteurs relativement indépendants. En effet, Anderson présente le «noble » de Lingga comme le chef des planteurs de poivriers de l'arrière-pays de Deli ${ }^{76}$.

L'introduction des plantations de poivriers, gambiers et cotonniers apporte à notre avis deux innovations fondamentales : le travail salarié et les migrations semipermanentes.

Ces cultures nécessitent une main-d'œuvre importante qui est ici autochtone à Sumatra-Nord et provient principalement des montagnes.

En 1823, jusqu'à deux jours de marche dans l'arrière-pays de Sunggal, Anderson prétend que pas moins de 20000 «Batak » de la «tribu Karo » sont engagés dans la culture. Il ne précise pas s'il s'agit exclusivement du poivrier mais ajoute qu'à la saison du poivre la rivière est presque impraticable en raison de la multitude de gens qui s'y rassemblent avec leur produit ${ }^{77}$.

Le poivre est en effet acheminé par les cours d'eau sur de petits sampans ou à dos d'hommes le long des sentiers.

De nombreux indigènes de l'arrière-pays laissent leur famille dans la montagne et descendent cultiver le poivrier, rentrant généralement chez eux une fois l'an avec le fruit de leur travail ${ }^{78}$. Ces cultivateurs quittant leur village un certain temps pour travailler contre une rémunération donnée à l'installation ou à l'entretien d'une plantation sont appelés dagang ${ }^{79}$.

A Sunggal, le dirigeant de la fédération avance à chacun d'eux, lors de son arrivée des montagnes, une certaine quantité de riz et une provision de sel pour un an ainsi que

70. Com. voor het Adatrecht, «Atjeh. Rechtsverhoudingen bij de pepercultuur (1913) », Adatrechtbundels, X, 1915 : 53-69.

71. Com. voor het Adatrecht, « Het maleische gebied. Rechtsverhoudingen bij de pepercultuur (1913) », Adatrechtbundels, X, 1915a : 253-258.

72. Anderson, $1826: 61,86,260$.

73. Anderson, $1826: 117$.

74. Mailrapport $818 / 1872$.

75. Netscher, $1864: 348$.

76. Anderson, Acheen and the Ports on the North and East Coast of Sumatra, London, $1840: 189$.

77. Anderson, $1826: 258$.

78. Anderson, $1826: 61$.

79. Dagang indique en fait une personne qui quitte sa maison pour aller gagner sa nourriture. 
les instruments nécessaires à la culture. L'opération est répétée pendant trois années successives. L'entrepreneur obtient les deux tiers de la récolte de poivre à prix réduit et le tiers restant au prix du marché ${ }^{80}$.

Ce flux migratoire donne naissance à des lieux de résidence collective, dusun ou kuta dagang, permettant à la main-d'œuvre de rester à proximité des terres à cultiver pour la production commerciale. Le kuta dagang se compose la plupart du temps d'une seule grande maison habitée exclusivement par des hommes ${ }^{81}$. Il subsiste dans la région près d'une dizaine de toponymes composés avec « dagang » (carte p. 167), dont un kuta dagang au sud de Sibolangit, au cœur du piémont. Ce type d'habitat est également utilisé dans d'autres plantations commerciales comme le gambier ${ }^{82}$.

En compensation de l'autorisation accordée par les « chefs » du plateau d'aller travailler dans les plantations, ces derniers prélevaient sans doute une bonne partie des revenus amassés par leurs «sujets ». Nous basons cette hypothèse sur une observation faite par le contrôleur Westenberg à Simalungun ${ }^{83}$. Les « chefs » du plateau ne pouvaient en effet accepter de laisser partir sans contrepartie des individus travaillant habituellement leurs propres terres ou élevant leur bétail. La rentabilité sans doute plus importante des plantations a permis cette migration observée par Anderson.

Il faut ajouter à cette main-d'œuvre issue du piémont et de l'est du plateau un apport d'esclaves originaires des rives du lac Toba. C'est surtout la zone au sud-est du lac qui apparaît comme la principale pourvoyeuse. C'est en effet dans la bande côtière voisine que semblent résider les marchands les plus importants ${ }^{84}$, intermédiaires dans un commerce qui doit concerner plusieurs milliers d'individus par an. Parmi eux figurent tout aussi bien le sultan d'Asahan que des Chinois, dont le rôle principal est d'approvisionner les mines d'étain de la péninsule malaise ${ }^{85}$.

Des esclaves de la même région arrivent aussi à Deli où le monopole de ce commerce semble être détenu par le sultan et dont les intermédiaires sont des marchands riverains de l'est et du nord du lac. Si beaucoup sont également envoyés en péninsule malaise ${ }^{86}$, on peut estimer à plusieurs centaines le nombre de ceux qui restent et travaillent pour les dirigeants de Deli ${ }^{87}$.

Anderson observe ainsi que le chef de la fédération de Sunggal, grand propriétaire de plantations, fait venir des esclaves d'Asahan dont il règle l'achat en poivre ${ }^{88}$.

\section{Plantations et catégories de populations}

Sur la côte nord-est, le processus d'islamisation (au moins depuis le Xve siècle) s'accompagne d'un processus d'acculturation sanctionné par l'adoption de l'identité

80. Anderson, $1826: 260-261$.

81. Joustra, 1902a : 423 .

82. Joustra, $1907: 126$; DPRD, $1977: 588$.

83. Westenberg, « Bataksche rijkes Dolok en Poerba », TNAG, XXII, 1905 : 597.

84. C'est peut-être dans cette région qu'il faut situer le pays d'Arcat réputé au début du $\mathrm{XVI}^{\mathrm{e}}$ siècle pour son marché aux esclaves (cf. Cortesao [éd.] et [trad.], 1944 : 148). Mais Arcat peut également être une déformation de Arkan, un toponyme utilisé pour Rokan sur la côte est face à Malaka dans les sources arabes anciennes (cf. Tibbetts, $1979: 248$ ).

85. Schadee, 1919, T.1: 130.

86. Sinar, communication personnelle (1990).

87. Cette estimation est faite à partir du nombre d' esclaves « affranchis » peu après l'arrivée du premier contrôleur Néerlandais (cf. Cats Baron de Raet, «Vergelijking van de vroegeren toestand van Deli, Serdang en Langkat met der tegenwoordingen », TBG, XXIII, 1876 : 33). A Deli, Anderson observe des esclaves «batak » travaillant sur les embarcations de hauts personnages (cf. Anderson, $1826: 89$ ).

88. Anderson, $1826: 77$. 
malaise (peut-être depuis le $\mathrm{XVI}^{\mathrm{e}}$ siècle) dont les traits sont clairement définis par les intéressés eux-mêmes : musulman parlant malais et suivant l'adat malais.

Sans affirmer que toute adoption de l'identité malaise est stratégique, il faut indiquer qu'elle peut comporter au moins trois avantages. Elle évite tout d'abord d'être vendu comme esclave. Il semble en effet que dans la région, un esclave qui se convertit à l'islam reste esclave mais ne puisse faire l'objet de transactions commerciales ${ }^{89}$. Elle permet d'autre part d'exploiter la terre pour son propre compte à la suite d'un processus qui est généralement le suivant : épouser une Malaise puis s'installer pendant trois à cinq ans dans un village malais où l'on s'ajuste avec la langue et l'adat malais. L'évolution est jugée par la communauté. Lorsque les conditions sont remplies, le chef du village demande au dirigeant de la fédération de donner de la terre au nouveau sujet. Enfin, en s'installant près de l'embouchure des rivières, un chef de l'arrière-pays peut alors, en prenant l'identité malaise, obtenir l'appui de l'élite côtière et en raison de son emplacement avantageux, rendre plus ou moins dépendantes de lui des localités situées en amont.

L'adoption de l'islam et d'une identité « étrangère » devrait impliquer en principe, par son caractère individuel, la suppression de toute référence à l'identité passée. Or, si elle se traduit effectivement par le choix d'un nom musulman et la disparition du nom du groupe patrilinéaire d'origine, elle n'entraîne pas une rupture fondamentale avec le reste de la population autochtone. Elle représente plutôt pour l'élite indigène des sultanats le moyen de devenir médiateur entre le monde extérieur et l'arrière-pays.

Le développement du sultanat grâce aux plantations instaure en fait un clivage politico-religieux dans la mesure où il se fonde sur l'islamisation d'une nouvelle classe d'autochtones influents qui s'enrichissent et vont progressivement remettre en cause l'influence politique dont pouvaient bénéficier jusque-là les « autorités » païennes du plateau sur leurs colonies respectives dispersées dans le piémont et la plaine côtière.

Mais, si les élites côtières malaïsées issues de l'arrière-pays détiennent le monopole du commerce international, leur ascension n'est rendue possible que grâce à l'existence de liens généalogiques avec l'arrière-pays.

Parallèlement sur le plateau, une élite probablement constituée par les descendants de ceux qui assuraient autrefois le bon fonctionnement des portages de produits forestiers vers la Côte Est, voit son statut renforcé par l'apport de compensations financières liées à l'expansion des plantations indigènes.

Au-delà de cette sphère malaise consciente de son unité culturelle mais aussi de son appartenance à une communauté plus large, évoluent des populations considérées comme un gisement humain pour l'esclavage, la main-d'œuvre des plantations et les épouses. On peut alors concevoir comment l'arrière-pays païen, ignorant des manières «civilisées » et de l'islam, va jouir progressivement d'une réputation d'infériorité en étant qualifié de «batak » par les Malais qui sont les exploiteurs de ces richesses.

L'examen de 1'organisation des plantations montre que l'existence du « clivage » Batak/Malais, traditionnellement perçu comme le signe d'une opposition entre deux groupes ethniques, masque en réalité un système cohérent où populations côtières et populations de l'arrière-pays sont étroitement liées.

\section{Adaptations et réactions aux plantations occidentales}

Lorsqu'il débute sa plantation en 1863, Nienhuys ne parvient pas à enrôler des autochtones. L'idée s'ancrera alors rapidement dans l'esprit des planteurs que ceux-ci

89. De Scheemaker, 1869a : 472. 
ne sont que des paresseux qui ne peuvent se tenir au travail ${ }^{90}$. Nous venons de voir que la réalité est différente et il est aisé de comprendre que déjà impliqués depuis longtemps dans les plantations indigènes, les autochtones hésitent à quitter soudainement le système traditionnel.

En effet, bien que manquant de témoignages directs durant les quarante années qui séparent le passage d'Anderson de la visite de Netscher, le Résident de Riau, il n'y a pas de raisons de penser que le système agricole se soit fondamentalement transformé.

Les exportations de poivre semblent toutefois moins abondantes qu'en $1823^{91}$. Les Hollandais attribuent cette baisse à l'extinction des plants et aux troubles continuels dans l'arrière-pays, troubles durant lesquels de nombreuses plantations auraient été ravagées. Dans le même temps, d'autres plantations se sont développées, notamment le muscadier et le cocotier ${ }^{92}$.

Avec l'installation du premier contrôleur hollandais à Deli en 1864 et sans doute stimulés par les expériences occidentales, les dirigeants locaux « affranchissent » un grand nombre d'esclaves qui vont, moyennant des accords garantissant des prix fixes, planter muscadiers et cocotiers. Ainsi vers 1865 , deux de ces dirigeants ont déjà fait planter des milliers de cocotiers et plusieurs centaines de muscadiers ${ }^{93}$.

Cette même année 1865 Nienhuys signe la première concession foncière avec le sultan de Deli ${ }^{94}$. En 1868 , Deli possède déjà six grandes plantations qui produisent plus de 200 tonnes de tabac ${ }^{95}$. Stimulé par ces résultats, Nienhuys crée l'année suivante la fameuse Deli Maatschappij qui, à sa fondation, couvre déjà 7000 hectares ${ }^{96}$ et se concentre principalement sur la culture du tabac. Un an plus tard, elle emploie 1200 coolies chinois sur un total de 3000 que compte le sultanat ${ }^{97}$ et rapidement le besoin en main-d'œuvre intensifie les liens avec la péninsule malaise, notamment Penang et Singapour.

Jusqu'à 1869 , les concessions accordées se trouvent uniquement dans la zone sous l'autorité directe du sultan.

Peu après, une première requête est faite pour l'obtention d'une concession dans la fédération de Sunggal. Elle sera à l'origine de ce que les Hollandais vont appeler la « guerre Batak», au moment où la région comprend déjà vingt-deux plantations de tabac ${ }^{98}$.

90. «And the Dutchman established the first tobacco plantation. The terrain he got from the radsha, but the natives would not work. The Malays of the forests had never done any work in their lives. They did not know how to work, and did not wish to learn. » (Székely, Tropic Fever. The Adventures of a Planter in Sumatra, Singapore, Oxford University Press, 1989 [1 re éd. 1937] : 62).

91. Anderson indiquait en effet pour Deli 26000 pikul, soit 1300 tonnes. Ces données de 1862 paraissent contredire les statistiques des importations de Penang qui indiquent un boom de la production poivrière sur la Côte Est jusqu'en 1859. Mais il est possible que cette expansion concerne presque uniquement le sultanat de Langkat.

92. Netscher, 1864 : 342, 344 ; Cats Baron de Raet, « Reize in de Battaklanden in December 1866 en Januarij 1867 »,TBG, XXII, 1875 : 172. La culture du muscadier n'est pas mentionnée par Anderson.

93. Cats Baron de Raet, $1876: 33$.

94. 2000 bouw (1 bouw =0,7 hect.) à Klumpang (cf. Dootjes, Deli-data (1863-1938), Mededeelingen $n^{\circ} 26$ van het Oostk. van Sum. Inst., Amsterdam, $1938: 5$ ).

95. Veth, $1877: 160$.

96. Pelzer, Planters and Peasant, Colonial Policy and the Agrarian Struggle in East Sumatra (1863-1947), VKI 84, 's Gravenhage, Nijhoff, 1978 : 38-39.

97. En 1870, les plantations emploient aussi déjà plus d'une centaine de Javanais, venus de Semarang (cf. Veth, $1877: 161$ ).

98. Pelzer, $1978: 52$. 


\section{La révolte de Sunggal}

En 1872, lorsqu'éclate la « guerre Batak », presque tous les chefs de fédérations de villages des basses terres sont encore propriétaires de plantations de poivriers et emploient une main-d'œuvre venue de la montagne ${ }^{99}$. Ce n'est par conséquent pas un hasard si les troubles se déclenchent au moment où les Occidentaux tentent de s'implanter sur leurs territoires.

Les initiateurs de la révolte sont Datuk Jalil et Datuk Kecil, deux frères du dirigeant de la fédération de Sunggal. Datuk Jalil a épousé une fille d'un chef de territoire du sultanat de Langkat. Cette alliance lui permet d'entrer en relation avec le sultan de Langkat, duquel il reçoit un titre. Il s'installe alors à la frontière entre les sultanats de Deli et de Langkat, sur un territoire revendiqué comme possession par chacun des sultans. Datuk Jalil joue rapidement sur cette position et l'hostilité entre les deux « monarques » pour se comporter en dirigeant indépendant ${ }^{100}$.

En 1869, le sultan de Deli essaie de reprendre autorité sur le territoire en donnant un titre au fils de Datuk Jalil, Sulung Barat. Sa soumission sera de courte durée. L'année suivante, accusé de comportements despotiques par Langkat, il refuse de répondre à une convocation du sultan de Deli. En 1871, soupçonné d'avoir saisi des marchandises appartenant à des planteurs, Datuk Jalil ne daigne pas rencontrer le Résident.

Lorsque le dirigeant de Sunggal meurt en 1857, il laisse un fils en bas âge. C'est l'autre frère, Datuk Kecil, qui se charge alors de l'administration de la fédération. Prenant goût au pouvoir il s'approprie tous les revenus sans remettre au futur datuk la part qui lui revient légitimement. Dès que l'héritier atteint l'âge requis, en 1866, le sultan de Deli décide de lui conférer le titre de Datuk Sri di Radja. Mais Datuk Kecil s'oppose à cette nomination, demandant notamment un partage des revenus de la fonction de datuk à son avantage.

Fermement opposé à la concession de terres par le sultan aux planteurs européens, il menace de brûler toutes les plantations de poivriers du datuk d'Hamperan Perak, le chef de la fédération voisine, au cas où celui-ci ose faire s'étendre à Sunggal les plantations occidentales qu'il a accueillies chez lui pour la première fois en $1870^{101}$.

Lorsque le premier planteur occidental fait une demande de concession au sultan pour des terres à Sunggal, Datuk Sri di Radja accepte dans un premier temps puis, sous la pression de son oncle, commence à montrer moins d'empressement. Datuk Kecil choisit alors de rejoindre son frère pour commencer la révolte en se retranchant dans des fortins ${ }^{102}$.

Fin mars 1872, un groupe de quarante indigènes armés menacent des coolies d'une plantation européenne occupés à ouvrir un chemin près de Langkat en avançant pour prétexte que la terre appartient aux « gens de la montagne ». Le lendemain, un autre planteur de Deli apprend par ses coolies chinois que le datuk de Sunggal vient de leur proposer une alliance pour attaquer les Européens ${ }^{103}$.

Quelques jours plus tard, le sultan de Deli fait part de son inquiétude au contrôleur hollandais installé à Labuhan Deli. Arguant des problèmes liés à cette situation, notamment les difficultés d'approvisionnement en riz et l'insécurité du territoire, il demande l'intervention de l'armée hollandaise pour venir à bout de la rébellion. Renseignements pris, les Hollandais estiment la force ennemie retranchée dans les fortins à deux jours et demi de Labuhan Deli à un minimum de mille «Batak » et cinq cents

99. Mailrapport 312/1872 ; Mailrapport 542/1872 ; Mailrapport 818/1872.

100. Mailrapport 312/1872; Mailrapport 823/1872.

101. Mailrapport 312/1872 ; Mailrapport 823/1872.

102. Mailrapport $823 / 1872$.

103. Res. Schift à GG 01/1873. Geh. en Kab. B23/1874. 
Malais armés de fusils et d'armes blanches. Le sultan ne peut quant à lui compter que sur une troupe de soutien d'environ deux cents hommes en qui il n'a toutefois pas trop confiance ${ }^{104}$.

La première expédition a lieu mi-mai 1872. La capture rapide des chefs de la rébellion s'avérant difficile, les Hollandais adoptent une tactique qui consiste à les obliger à se réfugier sur le plateau, où ils espèrent que les chefs en principe hostiles à la révolte les captureront et les remettront au Gouvernement. Celle-ci semble réussir puisque dans le courant du mois de juin, on apprend que les datuk se sont enfuis vers la montagne.

Début juillet, plusieurs fermes de séchage du tabac appartenant à des plantations occidentales sont brûlées. Quelques jours plus tard, les plantations de poivriers des insurgés, source principale de leur financement, sont détruites par l'armée ${ }^{105}$. Les troupes se déploient ensuite sur plusieurs plantations occidentales, ce qui n'empêche pas des attaques sporadiques des rebelles.

Les espions du sultan sillonnent l'arrière-pays et rapportent mi-juillet l'information selon laquelle les deux datuk et Sulung Barat se sont retranchés sur leur territoire en compagnie de trois chefs du plateau, Cuma Raja, Gajah et Bakti, et d'une troupe de 800 hommes dont 200 venus du plateau ${ }^{106}$.

Les militaires décident de passer à l'action fin septembre et finalement les chefs de l'insurrection se rendent le 25 octobre 1872 et sont immédiatement envoyés à Riau ${ }^{107}$ avant d'être bannis vers Java ${ }^{108}$.

L'analyse de cet épisode de la colonisation que constitue la révolte de Sunggal montre qu'il ne s'agit pas d'une guerre « ethnique », mais qu'elle allie la revendication d'autonomie de certains dirigeants, l'affirmation de droits fonciers et la défense d'un système traditionnel face au sultan.

Datuk Jalil et Sulung Barat se révoltent pour défendre l'autonomie qu'ils avaient commencé à acquérir vis-à-vis des sultans, montrant ainsi la fragilité de leur pouvoir et la puissance des chefs de fédérations.

Datuk Kecil agit quant à lui pour d'autres raisons. Son mobile nous paraît avant tout économique et politique. Il est le premier à ressentir une frustration liée au fait que le sultan s'approprie tous les revenus découlant de la présence des plantations européennes et se permette de concéder des terres situées dans les fédérations de villages. En effet, si le principe des taxes d'importation et d'exportation ainsi que la ferme de l'opium au profit du sultan étaient déjà en vigueur avant l'arrivée des Hollandais, le revenu tiré de ces droits a en revanche considérablement augmenté depuis l'installation des plantations occidentales. Il en est de même, mais dans une moindre mesure, des revenus du datuk d'Hamperan Perak. Le datuk de Sunggal ne bénéficie par contre que d'une augmentation sensible des droits attachés au commerce de l'opium.

Incapable de faire naître un soulèvement général en s'appuyant sur les autres chefs de fédérations de Deli, Datuk Kecil ne trouve finalement du soutien que dans son groupe généalogique sur le plateau. On sait en effet que le datuk de Sunggal, donc également ses frères Datuk Kecil, Datuk Jalil et le fils de ce dernier, Sulung Barat, sont issus du groupe patrilinéaire Lingga, tout comme les principaux chefs du plateau qui les soutiennent, à savoir les raja de Gajah, Surbakti et Cuma Raja qui tous trois sont originaires de la fédération de Telu Kuru ${ }^{109}$. L'hypothèse que nous avions émise, à propos

104. Mailrapport 312/1872.

105. Mailrapport 542/1872.

106. Mailrapport $636 / 1872$.

107. Mailrapport $793 / 1872$.

108. Veth, $1877: 164$.

109. Les femmes de Datuk Kecil et de Sulung Barat se réfugieront d'ailleurs avec leurs enfants à Gajah (Mailrapport 818/1872). 
de l'époque précédant l'arrivée des Hollandais, sur la primauté des espaces généalogiques unissant un urung de l'est du plateau à un urung de la côte, apparaît par conséquent encore valide en 1872. L'appellation « guerre Batak » nous semble encore moins justifiée dans la mesure où la mobilisation ne se fait pas sur des critères identitaires de ce type. En effet, si les datuk se disent Malais, les chefs de l'arrière-pays sont considérés comme «Batak ». Nous avons vu d'autre part, que les troupes recrutées appartiennent aux deux catégories.

Cet épisode de la colonisation fournit la preuve que les témoignages occidentaux doivent être considérés avec la plus grande prudence. Leur vision simplificatrice et la difficulté de compréhension masquent une réalité sociale complexe que seule une perspective historique nous a permis de mettre en évidence.

\section{Le détournement du réseau traditionnel de main-d'œuvre à partir de 1873}

Un mouvement certainement déjà amorcé avant la révolte de Sunggal prend de l'ampleur à partir de 1873. Il s'agit de l'embauche par les plantations occidentales de main-d'œuvre autochtone. Ainsi en 1873, la compagnie Deli Maatschappij emploie environ 350 individus au défrichage de la forêt pour les plantations de tabac, à la construction de baraques, à l'entretien des plantations de bananiers et de muscadiers et au transport du matériel en sampans ${ }^{110}$.

Lorsque, en 1876, Brau de Saint Pol Lias cherche à ouvrir une plantation dans la principauté de Bedagai, un individu se présente muni d'une lettre timbrée du sceau de son frère qui n'est autre que le dirigeant local, afin de proposer l'emploi de coolies « batak », qui sous ses ordres et sa responsabilité, pourraient mettre en place la plantation de tabac ${ }^{111}$. Nous savons que l'année suivante une plantation de tabac voisine ne possède pas moins de quarante «Batak » ou Malais sur une main-d'œuvre totale de 260 coolies ${ }^{112}$.

A Sunggal en 1880, le Français observe que les « anthropophages descendent de leurs montagnes, attirés par les dollars des Européens ». Ils viennent offrir leurs services pour la construction des cases et des séchoirs. Ils ne s'engagent jamais isolément, mais toujours par groupes plus ou moins nombreux sous la direction d'un « chef de leur race » et ne se mêlent ni aux Chinois, ni aux Malais ${ }^{113}$.

En 1881, Hagen cite l'exemple d'un homme riche et estimé de l'arrière-pays qui fait travailler des centaines d'individus dans les plantations occidentales ${ }^{114}$.

Deux ans plus tard, ce sont des milliers de personnes qui descendent de la montagne pour travailler dans les plantations occidentales ${ }^{115}$. Cette migration temporaire est non seulement le fait de gens du commun, mais celui de hauts personnages de l'arrière-pays, qui viennent tenter d'amasser une petite fortune afin d'accrôitre leur notabilité une fois rentrés chez eux. On voit se tisser des connexions entre chefs ayant déjà une expérience des plantations occidentales et ceux qui veulent se lancer dans l'aventure ${ }^{116}$.

110. Veth, $1877: 167$.

111. Brau de Saint Pol Lias, « Deli et les colons-explorateurs français », BSGP, 1877a : 305.

112. Brau de Saint Pol Lias, «Les colons explorateurs. Communication faite à la Société de Géographie commerciale le 26/10/1877 », L'Exploration, 4, 1877b : 152.

113. Brau de Saint Pol Lias, De France à Sumatra par Java, Singapour et Pinang. Les anthropophages., Paris, H. Oudin, $1884: 304$.

114. Hagen, «Rapport über eine im Dezember 1883 unternommene wissenschaftliche Reise an den Toba-See », TBG, 31, $1886: 328$. 146.

115. Hagen, "Zu den Wanderungen der Battas », Das Ausland 1883b : 11 ; Hagen, 1883a : 42,

116. Westenberg, $1897: 65$. 
Dans sa migration, le personnage influent emmène ses propres sujets, et c'est lui seul qui négociera avec l'administrateur de la plantation. Dès qu'ils ont trouvé du travail, ils bâtissent une maison à l'écart où vivent collectivement de cinquante à soixante hommes, ce qui n'est pas sans rappeler les kuta dagang de la période précoloniale. La plupart du temps, on leur alloue un petit champ où ils vont cultiver du riz qui est livré après récolte à la plantation contre paiement. Lorsque le travail est achevé, habituellement en août ou septembre, ceux qui estiment avoir assez d'argent retournent sur le plateau et sont remplacés par d'autres. En 1883, on estime que un quart à un tiers des hommes habitant sur la rive nord-est du lac ont déjà pris le chemin de Deli dans ce but ${ }^{117}$.

Il est aussi des cas où le raja ne descend pas vers les plantations, mais accorde à certains de ses sujets l'autorisation d'aller chercher du travail sur les plantations occidentales. Dans ce cas, les migrants sont hiérarchisés en coolies, mandur et kepala. Les mandur possèdent un certain prestige en raison de leurs liens de parenté avec le raja et le kepala, choisi parmi eux, est celui qui est chargé du recrutement, de la négociation avec l'entrepreneur et du bon déroulement du travail ${ }^{118}$. Il perçoit un pourcentage variant entre 5 et $10 \%$ sur les rémunérations des coolies ${ }^{119}$ et au retour, le raja récupère une bonne partie du solde ${ }^{120}$.

Le mouvement ne fait que s'accroître par la suite et les gens de l'intérieur acquièrent une solide réputation de défricheurs et de constructeurs de bâtiments en bois ${ }^{121}$. Ils se chargent également de la récupération des coolies évadés, organisant à cet effet de véritables battues qui, en cas de réussite, leur permettent d'empocher quelques guilders par individu récupéré ${ }^{122}$.

Dans les années 1930, des autochtones originaires de l'arrière-pays seront même employés comme coolies par les Occidentaux dans les plantations d'hévéa à Serdang ${ }^{123}$. Ces planteurs ont sans doute mis à profit une expérience similaire menée quelques vingt-cinq années plus tôt par le sultan.

Enfin, il faut signaler que, au moins depuis le milieu des années 1920 , nombreux sont les Malais à travailler comme journaliers sur les plantations de tabac, surtout les femmes et les enfants. Ils y sont employés principalement au ramassage des chenilles ou au tri du tabac ${ }^{124}$.

Contrairement à l'idée couramment admise, la main-d'œuvre autochtone, bien sûr largement inférieure en nombre aux coolies chinois et javanais participe donc massivement et activement à l'expansion des plantations occidentales.

117. Hagen, $1883 \mathrm{~b}: 11$.

118. « Their mandur [des coolies karo] was a powerful chieftain at home in the black mountains, here he was a powerful labour leader : a kapala orang » (Székely, 1989:122).

119. Tichelman, « Bataksch arbeidreservaat », KT, XXV, 1936a : 402-403.

120. Westenberg, 1905 : 597 ; Székely, 1989: 124 ; Ruiter, « Differentiation, Kinship and Conflict Resolution in a Lowland Karo Batak Village in North Sumatra », Paper presented at the 8th EMICS, Gothenburg, Sweden, 15-20/06/1991: 8.

121. Brenner J.F. von, Besuch bei den Kannibalen Sumatras: Erste Durchquerung der unabhängigen Batak-Lande, Würzburg, Woerl, 1894 : 18 ; Kroesen J.A., « Eene reis door de landschappen Tandjoeng Kassau, Siantar en Tanah Djawa », TBG, XXXIX, 1897 : 254 ; Bernard, A travers Sumatra, Paris, Hachette, 1904 : 188 ; Kroesen et Helderman, 1904 : 580 ; Naudin ten Cate et Wall Perne, Deli in woord en beeld, Amsterdam, De Bussy, 1905. Il arrive également qu'une plantation s'installe dans le dusun, emploie un certain nombre d'individus des villages proches comme coolies et les chefs de ces villages comme surveillants (cf. Joustra, "Verslag van de zending onder de Karo-Bataks, over het jaar 1895 », MNZ, 40, 1896 : 226).

122. Cf. notamment Székely, $1989: 141-142$.

123. Gerritsen, Mvo Serdang, $1938: 38$.

124. Ruychaver, Mvo Beneden Deli, 1926 : 37. 
Si ces dernières entrent rapidement en concurrence avec les autochtones pour la possession de l'espace, nous voyons que naît également une concurrence pour l'appropriation de la main-d'œuvre de l'arrière-pays, seule capable d'effectuer certaines tâches. Cette concurrence est certainement d'autant plus âpre que l'interdiction de l'esclavage empêche les autochtones de se procurer une main-d'œuvre à l'extérieur comme ils le faisaient avant l'arrivée des Occidentaux.

\section{Le sort des entrepreneurs autochtones}

Les sultans et leurs proches profitent de la présence des planteurs occidentaux pour développer leur patrimoine personnel de plantations en adaptant l'organisation traditionnelle des cultures rebaptisée dagangstelsel par les Hollandais.

En 1876 le frère du sultan de Deli administre 700 hectares de plantations aux environs de Deli Tua ${ }^{125}$.

On sait que vers 1880 , le sultan de Serdang possède de belles plantations de caféiers à Pertumbukan ${ }^{126}$. En 1910, il commence une plantation d'hévéa, obligeant les autochtones, lorsqu'ils en ont terminé avec les récoltes sur leur ladang, à planter des hévéas sur ces mêmes ladang. Ceux-ci se trouvant tout près des villages, les abords immédiats sont ainsi transformés en plantations.

Les fonctionnaires hollandais voient d'un mauvais œil ce système traditionnel indigène, mettant l'accent sur les pouvoirs exorbitants des chefs et de leurs subordonnés qui inventent toutes sortes de stratagèmes pour spolier les dagang. Cette affaire du sultan de Serdang est portée devant le Résident qui lui signifie que les fonctions de sultan et de planteur sont incompatibles ${ }^{127}$. Il semble toutefois qu'aucune décision gouvernementale ne soit prise pour sanctionner le dirigeant de Serdang puisque la plantation existe toujours en 1932 et emploie 387 personnes ${ }^{128}$. A la même époque, il possède une grande plantation de tabac ${ }^{129}$ ainsi qu'une grande plantation de cocotiers couvrant environ 385 hectares pour 13000 arbres ${ }^{130}$.

A Deli, les chefs de fédérations paraissent connaître des fortunes diverses. C'est surtout celui d'Hamperan Perak qui semble tirer un profit maximum de la présence des planteurs occidentaux. Nous avons signalé précédemment qu'il est le premier datuk à avoir accueilli des plantations occidentales sur son territoire. De plus, disposant dès avant l'arrivée des Hollandais du monopole sur le commerce du nipah, il va profiter du boom induit par le développement des plantations pour accroître ses revenus de manière significative ${ }^{131}$. Les palmes du nipah servent en effet à la confection des toits et des cloisons de baraquements mais aussi et surtout à celle des fermes de séchage du tabac.

En 1877, Hamperan Perak dispose encore de plantations de poivriers certainement possédées en bonne partie par le datuk ${ }^{132}$. En 1899 , ce dernier commence l'exploitation

125. Veth, $1877: 166$

126. Hagen, 1883a : 42. Victimes de maladie, elles disparaîtront en 1883 (Hagen, $1886: 330$ ).

127. Kok, Mvo dusun Deli-Serdang, $1910: 80-81,150,151$.

128. De Ridder, Mvo Serdang, 1933 : 29. Au moins dès les années 1910, le sultan de Serdang possède également environ 900 bouw de sawah près de Perbaungan, que la population peut utiliser moyennant redevance. Il est propriétaire d'autre part d'un cheptel d'environ 400 buffles (van Kempen, Mvo Serdang, 1912 : 22-23 ; Reuvers, Mvo Serdang, $1923: 20$ ).

129. Le tabac récolté servait à la fabrication de cigares portant la marque et l'image du sultan de Serdang Sulaiman (Ezerman, Mvo x/S.O.K., 1933 : 6; Sinar, 1986, T.2 : 30). Cette plantation sera fermée en 1934 (Gerritsen, Mvo Serdang, $1938: 40$ ).

130. De Ridder, Mvo Serdang, 1933 : 29 ; Gerritsen, Mvo Serdang, 1938 : 39.

131. Pelzer, $1978: 114$.

132. Veth, $1877: 158$. 
de plantations de caféiers dans le piémont de Deli, à la limite du plateau. Elle sont dirigées par son beau-frère, métis européen, assisté de deux Européens ${ }^{133}$.

En 1880 à Sunggal, il y a toujours de nombreuses plantations de poivriers entretenues par des autochtones ${ }^{134}$, ce qui laisse à penser que le datuk poursuit son activité. En 1883, Hagen traversant le piémont constate que les plantations de poivriers qu'il avait pu observer deux années plus tôt ont disparu ou sont à l'abandon, remplacées par des rizières et des champs de maïs ${ }^{135}$.

Il semble qu'à côté de la baisse des cours ${ }^{136}$ les planteurs occidentaux aient fortement contribué à ce déclin des plantations autochtones de poivriers dans les sultanats de Deli et Serdang, notamment en interdisant la création de nouvelles et le remplacement de celles qui s'épuisent. Il en sera de même pour les plantations autochtones de tabac.

La culture du poivrier cède devant les grandes plantations occidentales et se réfugie pour un temps à leur périphérie.

Si à Deli et Serdang elle semble en effet décliner fortement à partir des années 1880 et être quasiment abandonnée à la fin des années 1910, elle se poursuit activement à Langkat, au moins dans sa partie nord où vivent de nombreux Acihais ${ }^{137}$. Il en est de même à Simalungun où ces plantations semblent connaître un essor significatif entre 1890 et $1910^{138}$.

Quant aux plantations traditionnelles de cotonniers, elles ont déjà pratiquement disparu lorsque les Occidentaux s'installent.

Il faut mentionner également l'intervention des Chinois dans les nlantations indigènes, notamment les plantations de muscadiers. En effet, dès la fin du XIX ${ }^{\mathrm{e}}$ siècle, des dirigeants autochtones choisissent d'affermer leurs plantations de muscadiers où de vendre leur récolte de noix de muscade à des Chinois ${ }^{139}$.

Des plantations autochtones sont également développées par le biais des chefs de tarekat et des missionnaires protestants. En ce qui concerne les premiers, l'exemple le plus connu est celui de Syekh Abdul Wahab, chef important de la tarekat Naqsybandiyah qui, en 1882, fonde à Langkat le village de Babussalam ${ }^{140}$. Les missionnaires protestants du piémont de Deli et de Simalungun vont quant à eux mettre en place dans la première décennie du $\mathrm{XX}^{\mathrm{e}}$ siècle des «plantations paroissiales » (gemeentetuin) de rambung (ficus elastica) ${ }^{141}$.

133. Guillaume, « Karo-Batak Zending. Jaarsverslag aangaande het zendingsressort Boekoem », $M N Z, 45,1901$ : 125 ; Joustra, «Het jaar 1901 onder de Karo-Bataks », MNZ, 46, 1902b:61 ; Schadee, 1920, T.2 : 23. Cette plantation existe encore en 1922 (PewDeli 17/03/1922).

134. Brau de Saint Pol Lias, $1884: 384$.

135. Hagen, $1886: 330$.

136. Encyclopaedie van Nederlandsch-Indie, Leyden, M. Nijhoff, 1917, T.1 : 69.

137. Anonyme, « Aanvullingsnota van toelichting betreffende het rijk van Langkat », TBG, LIII, 1911: 328 ; ENI, 1919, T3 : 151; Broersma, Oostkust van Sumatra, Batavia/Deventer, Jav. Boekhandel./ C. Dixon, 1919-1922: 8.

138. Van Dijk, « Rapport betreffende de Sibaloengoensche landschappen Tandjoeng Kassau, Tanah Djawa en Si Antar », TBG, XXXVII, $1894: 161,165$; Kroesen, $1897: 260$; Kroesen, « Nota omtrent de Bataklanden », TBG, XLI, 1899 : 265 ; Simon, «Die Arbeit der rheinische Mission auf Sumatra Ostküste », $A M Z, 33,1906$ : 468 ; Anonyme, « Nota van toelichting betreffende de Simeloengoesche landschappen Siantar, Panei, Tanah Djawa en Raja », TBG, 51, 1909 : 549.

139. La production de noix de muscade va fortement chuter à partir du début du $x^{e}$ siècle en raison des maladies frappant les vieux plants et des cours trop bas (Broersma, 1919-1922, T.2: 8).

140. Fuad Said, Syekh Abd. Wahab, Tuan Guru Babussalam, Medan, Pustaka Babussalam, 1988 : 63, 67, 106, 107. Cf. également Lombard, «Tarekat et entreprise à Sumatra : l'exemple de Syekh Abdul Wahab Rokan (c. 1830-1926) », in M. Gaborieau ; A. Popovic et T. Zarcone (éd.), Naqshbandis. Actes de la table ronde de Sèvres, 2-4 mai 1985, Istambul/Paris, Isis, 1990 : 707-716.

141. Neumann, « De Deli-Zending. Ressort Sibolangit. Het jaar 1908 », MNZ, 53, 1909a : 69. 


\section{Conclusion}

Nous avons mis en évidence l'interdépendance qui existe sur la côte est de SumatraNord entre populations de l'intérieur et populations côtières au moins depuis l'apparition d'un commerce international dans la région, sans doute dès la fin du Ier millénaire de notre ère. Après avoir connu ce qui paraît être une grande période de prospérité entre le $\mathrm{XII}^{\mathrm{e}}$ et le XIVe siècle, la région de Deli est probablement délaissée par les réseaux commerciaux musulmans à la suite de la prise de Malaka en 1511. Jusqu'au XVIII ${ }^{e}$ siècle, elle semble plutôt jouer un rôle d'intermédiaire plus ou moins actif entre, d'une part, des marchands étrangers et, d'autre part, l'ouest de l'île riche en camphre et en benjoin, la partie centrale au nord du lac Toba qui fournit chevaux et riz, les zones à l'est et au sud du lac Toba pourvoyeuses d'esclaves et peut-être les régions méridionales de Minangkabau et Jambi pour la fourniture d'or.

La toponymie atteste la densité des lieux d'interactions qui sont supposées, pendant cette période, réglées par des accords ou serments. Les réseaux d'échanges sont alors caractérisés par des déplacements incessants de porteurs contrôlés dans l'arrière-pays par une élite assurant l'approvisionnement régulier de comptoirs dont l'importance et la localisation varient et où des individus s'approprient le monopole des échanges extérieurs.

Après une éclipse économique au profit du nord de l'île, la côte nord-est tire parti d'une situation confuse à Aceh et d'un regain de la demande mondiale pour retrouver une importance économique en développant des plantations de poivriers. La datation de l'apparition de ces plantations est une question délicate. S'il est certain que Langkat en exploite dès la fin du XVIII e siècle, il faut attendre 1823 pour avoir un témoignage irréfutable en ce qui concerne Deli.

L'identification des promoteurs est tout aussi difficile. Seules, des traditions locales nous mettent sur la piste d'individus originaires du centre de l'île ou de la côte ouest. Recoupées avec des données archéologiques et généalogiques, elles situeraient les premières plantations entre le XIVe siècle et la première moitié du XVIrre siècle.

Avec le développement concomitant des plantations de cotonniers et de gambiers, qui, comme nous l'avons vu, ont laissé leur nom aux espaces du piémont que sont les sinuan bunga et sinuan gambir, on assiste à Deli au début du XIX $\mathrm{X}^{\mathrm{e}}$ siècle, à la naissance d'une véritable « aire de culture » dont les toponymes ne reflètent plus qu'imparfaitement l'extension.

Le sultan de Deli, Amaluddin Panglima Mangedar Alam, apparaît, au début du $\mathrm{XIX}^{\mathrm{e}}$ siècle, comme l'instigateur principal de ces transformations. A l'origine d'un mouvement fédérateur des suku des basses terres, l'institution « Datuk Empat Suku », il réussit à multiplier les alliances pour s'assurer l'appui d'individus capables de mobiliser les espaces et la main-d'œuvre nécessaires à l'expansion des plantations.

Deux types d'exploitations semblent avoir coexisté au début du XIX ${ }^{\mathrm{e}}$ siècle : des " grandes plantations » détenues et contrôlées par le sultan et les chefs de fédérations qui, dans les parties basses, font travailler, moyennant rémunération en espèces, des migrants du plateau et des « esclaves »; des «plantations familiales » dans le piémont, contrôlées par des hauts personnages du plateau.

Si les réseaux commerciaux concernant les produits forestiers étaient caractérisés par des migrations temporaires, l'arrivée des plantations se traduit par l'installation des migrants dans des habitats collectifs, les kuta dagang qui, tout compte fait, ne sont que les précurseurs à échelle réduite des pondok des plantations occidentales dans lesquels s'entasseront les coolies chinois et javanais à partir de la deuxième moitié du $\mathrm{XIX}^{\mathrm{e}}$ siècle. 
Comme plus tard les planteurs occidentaux, les planteurs autochtones doivent faire face à une pénurie de main-d'œuvre. Celle-ci sera mobilisée non seulement dans l'arrière-pays jusque sur le plateau mais aussi également importée de territoires voisins du sud et de l'est du lac Toba, par l'intermédiaire du sultanat d'Asahan notamment. Ces « esclaves », introduits à Deli au moins depuis le début du XIX ${ }^{\mathrm{e}}$ siècle, précèdent ainsi de quelques décennies les coolies chinois importés de la péninsule malaise.

S'il fallait faire un autre parallèle avec la situation économique qui prévaudra un siècle plus tard, nous mentionnerons la pénurie en riz qui frappe la Côte Est au moment où elle développe ses plantations. De la même manière, fort accroissement de la population résidante et transformation des ladang en plantations en constituent probablement les causes principales.

En réalité, à la veille de la colonisation, le nord-est de Sumatra, et tout particulièrement l'ensemble formé par les sultanats de Langkat, Deli, Serdang, les régions au nord et à l'est du lac Toba, se présente comme un grand espace en voie de malaïsation dont la cohérence est fondée sur des liens généalogiques et une interdépendance économique. Tout en perdant le monopole de la médiatisation avec le monde extérieur, les sultans maintiennent leur légitimité grâce à la reconnaissance des Hollandais. Elle se traduit par un enrichissement exceptionnel qui est à notre avis un facteur de désarticulation avec l'arrière-pays. La guerre de Sunggal en est le premier symptôme.

Interprétée à tort comme une guerre « ethnique » par les témoins occidentaux, elle révèle en fait la primauté des liens généalogiques sur les catégories «Malais » et «Batak » qui seront pourtant progressivement réifiées par les colonisateurs. Le détournement de la main-d'œuvre ainsi que des élites du piémont et du plateau vers les plantations occidentales consacre quant à lui une rupture des liens économiques étroits entre urung de l'est du plateau et urung de la côte.

Il est permis de penser que cette forme d'indépendance vis-à-vis des dirigeants de la côte, qui permet de faire l'économie d'un intermédiaire pour tirer directement les bénéfices de relations avec le monde extérieur, a pour effet une prise de conscience par l'arrière-pays d'une liberté relative vis-à-vis de la côte et d'une importance en soi. Liée à la généralisation de la monétarisation des échanges par la création d'un espace monétaire pratiquement unifié, cette liberté nouvelle a très certainement encouragé également l'individualisme économique, d'autant plus que les modèles du genre ne manquent pas dans les basses terres. Celui-ci peut à son tour permettre l'émergence de nouvelles formes de solidarités.

Rapidement, les dirigeants indigènes perdent le monopole du contrôle et de l'expansion des plantations autochtones traditionnelles dont ils confient parfois l'exploitation à des Chinois. Religieux musulmans et protestants interviennent également en développant des plantations qui, au-delà de leur intérêt économique, visent au renforcement des solidarités confessionnelles et des sentiments communautaires à l'intérieur de nouveaux espaces que constituent par exemple les paroisses.

L' « aire de culture » autochtone traditionnelle disparaît pratiquement au début du $\mathrm{XX}^{\mathrm{e}}$ siècle pour céder la place aux plantations coloniales, et seuls les sultans et leurs proches, qui imitent les planteurs occidentaux, conservent ou renforcent leurs activités. 